\title{
Genus level molecular phylogeny of Aegisthidae Gisbrecht, 1893 (Copepoda: Harpacticoida) reveals morphological adaptations to deep-sea and plagic habitats
}

\author{
Sahar Khodami *iD, Nancy F. Mercado-Salas and Pedro Martìnez Arbizu
}

\begin{abstract}
Background: The family Aegisthidae is known as typical component of deep-sea hyperbenthic waters that gradually colonized other marine environments. The phylogenetic relationships within this family have been examined here including hyperbenthic, planktonic, benthic forms and two associated Aegisthidae species.

Results: Ninety four specimens belong to 14 genera were studied using 185 and 285 rRNA and COI mtDNA. Bayesian analysis supports the monophyly of 10 genera whereas Andromastax, Jamstecia, Nudivorax and Aegisthus revealed to be paraphyletic. The first offshoot of the phylogenetic tree is a clade consists of the undescribed genus Aegisthidae gen.1 sister to the two monophyletic genera Cerviniella and Hase, whereas the other Cerviniinae members (represented by Cervinia and Expansicervinia) assemble a monophylum, sister to the hyperbenthic and planktonic aegisthid genera, resulting in the paraphyly of the subfamily Cerviniinae. Hence, we defined the new subfamily Cerviniellinae subfam. nov. encompassing the three benthic genera Cerviniella, Hase and Eucanuella. The subfamily Cerviniinae has been re-defined to include Cervinia, Expansicervinia and Paracerviniella. Members of the subfamily Pontostratiotinae were clustered into two clades, one consists of the genus Stratiopontotes sister to an undescribed genus + Cerviniopsis and Siphonis. The second contains Pontostratiotes sister to the members of the planktonic subfamily Aegisthinae, resulting in the paraphyly of the Pontostratiotinae. Therefore, the Pontostratiotinae has been re-defined to include only members of the genus Pontostratiotes; whereas the subfamily Cerviniopseinae has been re-erected and re-defined containing Stratiopontotes, Cerviniopsis, Siphonis, Aegisthidae gen. 2, Herdmaniopsis, Hemicervinia and Tonpostratiotes. Within this subfamily, the associated Siphonis clusters as sister to the Cerviniopsis represents an example of convergent evolution in which the possession of a stylet-like mandible and an oral cone reminiscent of the Siphonostomatoida. The planktonic Aegisthus, Andromastax, Jamstecia, Nudivorax and Scabrantenna confirm the monophylom Aegisthinae, sister to the Pontostratiotinae.
\end{abstract}

Conclusions: Our DNA based phylogeny reveals the deep-sea origin of Aegisthidae by placing benthic Aegisthidae gen.1 and Cerviniellinae subfam. nov. as the most basal lineages. Secondary adaptations to hyperbenthic and planktonic realms, as well as associated lifestyle were discovered here by the derived positions of Pontostratiotinae, Aegisthinae and Siphonis respectively.

\footnotetext{
* Correspondence: sahar.khodami@senckenberg.de

Senckenberg am Meer, German Centre for Marine Biodiversity Research,

Südstrand 44, 26382 Wilhelmshaven, Germany
}

(c) The Author(s). 2020 Open Access This article is distributed under the terms of the Creative Commons Attribution 4.0 International License (http://creativecommons.org/licenses/by/4.0/), which permits unrestricted use, distribution, and reproduction in any medium, provided you give appropriate credit to the original author(s) and the source, provide a link to the Creative Commons license, and indicate if changes were made. The Creative Commons Public Domain Dedication waiver (http://creativecommons.org/publicdomain/zero/1.0/) applies to the data made available in this article, unless otherwise stated. 


\section{Background}

It is generally believed that, ancestral copepods were living in benthic or hyperbenthic habitats [1]. The pelagic realm was colonized later during the evolution of copepods by developing specialized A1 and thoracic appendages [2-4], in association with feeding and mating behavior [5-7]. The Harpacticoida Sars G.O., 1903 is a highly successful group of Copepoda Milne Edwards, 1840 in terms of speciation and adaptations to deep sea environments [8]. Within harpacticoids, the family Aegisthidae Giesbrecht, 1893 is often the dominant taxon of deep-sea hyperbenthic communities [9-15]. Giesbrecht (1892) established the family containing two species of the mesopelagic genus Aegisthus Giesbrecht, 1891 A. mucronatus Giesbrecht, 1891 and A. aculeatus Giesbrecht, 1891. Later Sars (1903) established the family Cerviniidae Sars, 1903 for some benthic and hyperbenthic species. Lang [16] proposed the taxon Cerviniidimorpha Lang, 1944 to unite the Cerviniidae and the Aegisthidae into a monophyletic taxon. Several studies have mentioned taxonomic problems within the family Aegisthidae (e.g. [17-21]) but the phylogenetic relationships among the genera remained uncertain. Seifried and Schminke [22] synonymized Cerviniidae with Aegisthidae and proposed a taxonomic system of the Aegisthidae divided into three subfamilies Aegisthinae Giesbrecht, 1893, Cerviniinae Sars M., 1903 and Cerviniopseinae Brotskaya, 1963. Later Huys [23] has synonymized the Cerviniopseinae with Pontostratiotinae Scott, A., 1909 based on the principle of the priority of names, however this new arrangement was not followed by many authors and this subfamily was commonly referred as Cerviniopseinae.

In a morphology-based phylogenetic study conducted by Seifried and Schminke [22], the families Aegisthidae and Rometidae Seifried \& Schminke, 2003 (= Aegisthoidea Giesbrecht, 1892) were considered as the most basal lineages of Oligoarthra Lang, 1944; however the evolutionary history within the family Aegisthidae was not studied.

Here, we attempt to resolve the phylogenetic relationships within this family based on a diverse collection of deep-sea aegisthid species inhabiting different habitats of the Atlantic and the Pacific oceans. Our molecular phylogeny of the Aegisthidae is based on small (18S) and large subunits (28S) of nuclear ribosomal RNA and the mitochondrial gene Cytochrome $c$ oxidase subunit I (COI). Sixty species representing 14 genera of the three subfamilies were chosen for our phylogenetic analyses. This study represents the first attempt to reconstruct the evolutionary history of this diverse family using molecular methods and includes numerous taxa, some of them phylogenetically important, in all currently recognized subfamilies. A brief discussion of the main morphological adaptations is provided.

\section{Results}

Bayesian phylogenetic inference using two nuclear (18S and $28 \mathrm{~S}$ rRNA) and one mitochondrial (COI mtDNA) gene sequences recovered Aegisthidae as a monophyletic clade. Ten monophyletic genera were recovered with high support values (posterior probabilities): Aegisthidae gen. 1, Cerviniella Smirnov, 1946, Hase Corgosinho, Kihara, Schizas, Ostmann, Martinez Arbizu \& Ivanenko, 2018, Cervinia Norman in Brady, 1878, Expansicervinia Montagna, 1981, Stratiopontotes Soyer, 1970, Siphonis Mercado-Salas, Khodami \& Martínez Arbizu, 2019, Cerviniopsis Sars G.O., 1903, Aegisthidae gen. 2, and Pontostratiotes Brady, 1883. Our phylogeny did not support the monophyly of four genera, Andromastax Conroy-Dalton \& Huys, 1999; Jamstecia Lee W. \& Huys, 2000, Aegisthus Giesbrecht, 1891 and Nudivorax Lee W. \& Huys, 2000. Among the three subfamilies previously assigned to the Aegisthidae [21, 22] only the Aegisthinae was recovered monophyletic, whereas, Cerviniinae and Pontostratiotinae were paraphyletic. The description of the new genus and species will be presented elsewhere. Figure 1 illustrates the phylogenetic tree of aegisthid species analyzed in this study in which five distinct clades have been revealed using Bayesian analysis:

\section{Clade 1}

Encloses the undescribed genus Aegisthidae gen. 1 sister to a monophylum containing the benthic Cerviniella and Hase. We proposed the new subfamily Cerviniellinae subfam. nov. enclosing the genera Cerviniella and Hase (included in the molecular analysis) as well as Eucanuella Scott T., 1901 (based on morphological apomorphies from previous studies). The undescribed genus (Aegisthidae gen. 1) has not been included in this subfamily following distinct morphological differences, therefore retained as a single independent lineage within the clade 1 . Figures 2 and 3 show confocal scanning images from habitus and mouthparts of the undescribed genus Aegisthidae gen. 1 and representatives of the subfamily Cerviniellinae (Cerviniella and Hase) respectively.

\section{Clade 2}

Contains four genera Aegisthidae gen. 2, Cerviniopsis, Siphonis and the genus Stratiopontotes in which the last genus was recovered sister to all remaining genera within this clade. The subfamily Cerviniopseinae has been reerected here to contain these four genera. Also Hemicervinia Lang, 1935, Herdmaniopsis Brotskaya, 1963 and Tonpostratiotes Itô, 1982 are recognized here as members of the re-erected subfamily Cerviniopseinae only based on morphological evidence. The associated genus Siphonis has shown to be a derived lineage of Cerviniopseinae sister to Cerviniopsis. Figure 4 presents scanned images from the genera Stratiopontotes and Siphonis. 


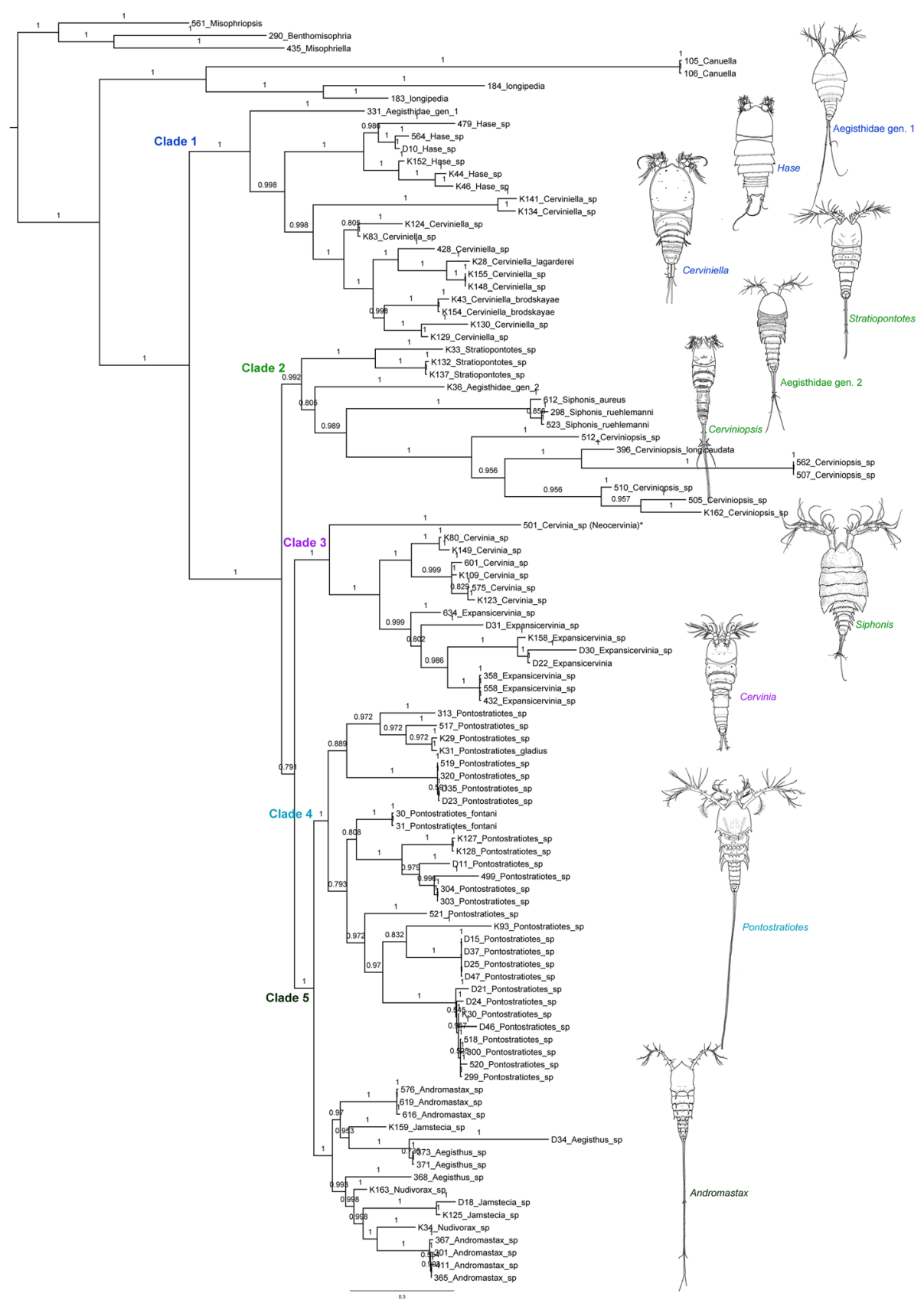

Fig. 1 Phylogenetic relationship of 94 specimens representing 14 genera of Aegisthidae based on Bayesian analysis of 18S, 28S rRNA and COI mtDNA. The five distinct clades are marked in the tree. Nodal supports are indicated by posterior probabilities. Scale bar shows nucleotide changes per site

\section{Clade 3}

A well-supported clade consists of two genera: Cervinia and Expansicervinia. Two distinct lineages were recovered within the genus Cervinia; one corresponds to the lineage previously known as Neocervinia Huys, Møbjerg \& Kristensen, 1997(synonymized with Cervinia by [21]) sister to the rest of the Cervinia. However the both lineages are treated here as Cervinia due to a single specimen of the
"Neocervinia-lineage" was available for this study. The re-validation of this taxon will be made elsewhere when enough material is available to support its taxonomic status. Here, the subfamily Cerviniinae is re-defined to enclose the genera Cervinia, Expansicervinia and Paracerviniella Brodsky, 1963 (the latest only based on morphological criteria). Figure 5 depicts the confocal scanning images form the representative of the subfamily Cerviniinae. 


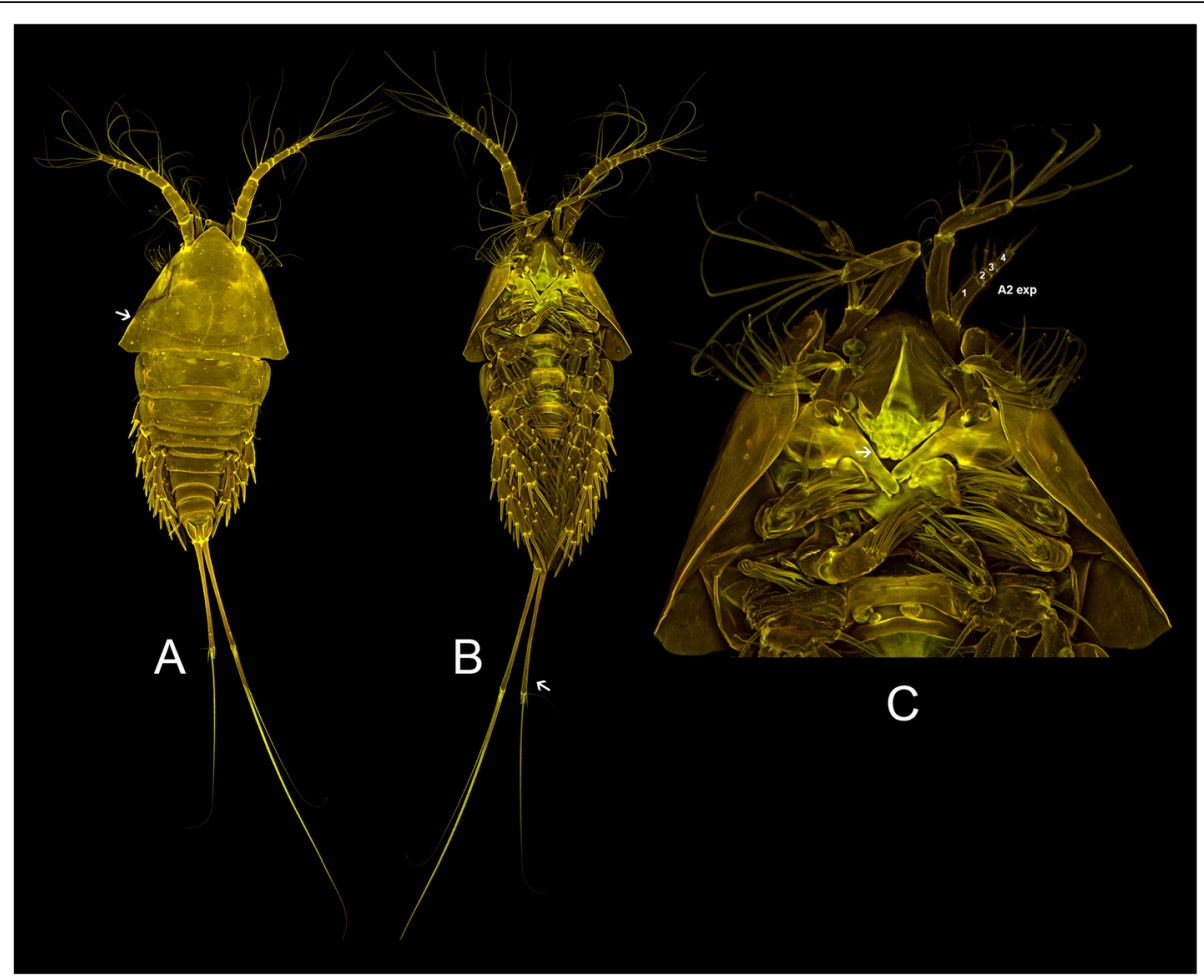

Fig. 2 Confocal laser scanning of the Aegisthidae gen. 1, habitus, dorsal (a), ventral (b) and mouthparts (c)

\section{Clade 4}

A highly supported monophyletic group including only members of the genus Pontostratiotes. Several lineages of Pontostratiotes were recovered which indicates the high diversity within the genus. Here we re-define the subfamily Pontostratiotinae as a monotypic taxon within Aegisthidae to accommodate species belonging to the genus Pontostratiotes only (Fig. 6d, e and f).

\section{Clade 5}

Contains the genera traditionally assigned to the subfamily Aegisthinae: Aegisthus, Andromastax, Jamstecia, Nudivorax and Scabrantenna Lee W. \& Huys, 2000 (Fig. $6 \mathrm{a}, \mathrm{b}$ and c). Although this clade is highly supported to be monophyletic, the four genera, as currently defined morphologically, (Andromastax, Aegisthus, Nudivorax and, Jamstecia) are paraphyletic. Scabrantenna is not available in this study.

Two offbeat tree topologies have been resulted here based on two different character (nucleotide) partition settings implemented in Bayesian phylogenetic analysis from two different independent runs (see methods). The second tree topology (Additional file 1: Figure S1) contrasts with the first tree in 1) the position of Aegisthidae gen. 1, being sister to the genus Hase, 2) Cerviniella is paraphyletic, Hase and the Aegisthidae gen. 1 are nested within Cerviniella 2) the subfamilies Cerviniinae clustered as sister to an unstable clade containing
Cerviniopseinae sister to Pontostratiotinae + Aegisthinae, 3) the genus Stratiopontotes is sister to a polytomy of Siphonis + Aegisthidae gen. $2+$ Cerviniopsis, 4) the topology of the inter-clades within the genus Pontostratiotes is different in some lineages. This alternative phylogram displayed extremely low EES (estimated sampling size) value for the Log-likelihood estimation ( $\mathrm{LnL}$ ), it shows significantly low supports for some phylogenetically important clades and is incongruent with the principle of parsimony as accepting this topology would imply the character reversal of the unique modification of swimming legs present in Cerviniella (arrow in Fig. 3d) but also of the apomorphies of Cerviniellinae subfam. nov. as discussed below, to a morphologically unmodified condition in Aegisthidae gen 1. (Fig. 2b); hence this topology is presented in the supplementary material but not considered here for further discussion. The information about both MrBayes jobs including the alignments, nexus blocks, generated trees and the MrBayes log files (information about the runs) are available as Job1 and Job2 supplementary information (Additional files 2 and 3).

\section{Discussions}

The monophyly of Aegisthidae was examined here for the first time using molecular data of $18 \mathrm{~S}, 28 \mathrm{~S}$ rRNA and COI mtDNA. Our result confirms the monophyly of the family Aegisthidae established by Seifried [21] and Seifried and Schminke [22] based on morphological 


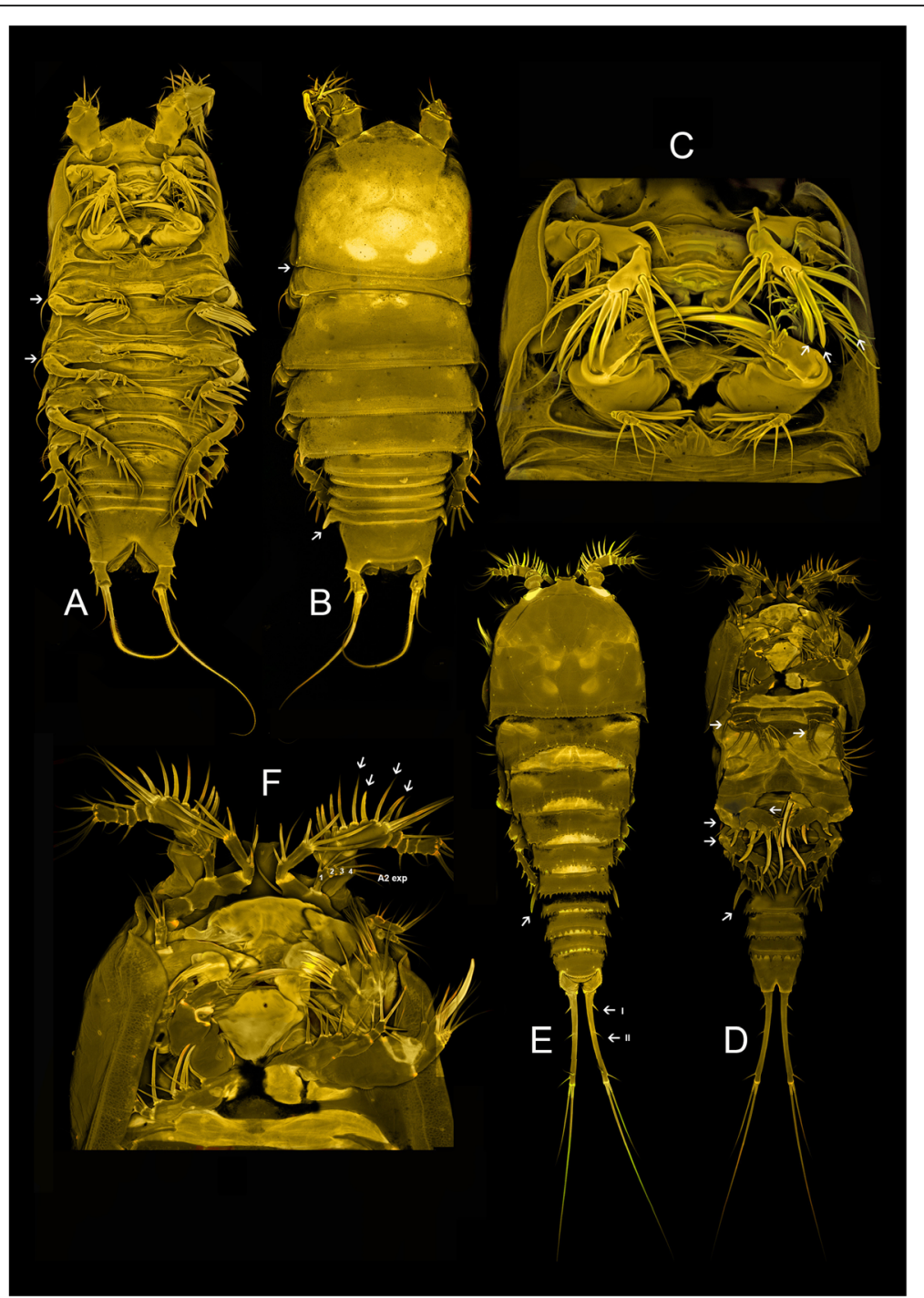

Fig. 3 Confocal laser scanning of the taxa representing subfamily Cerviniellinae subfam. nov. Hase talpamorphicus Corgosinho, Kihara, Schizas, Ostmann, Martinez Arbizu \& Ivanenko, 2018. habitus, dorsal (a), ventral (b) and mouthparts (c). Cerviniella sp. habitus, dorsal (d), ventral (e) and mouthparts (f). Images of Hase talpamorphicus are adapted from Corgosinho P.H.C et al. [24]

characters. The traditional morphological view that, consider three monophyletic subfamilies within Aegisthidae $[21,22,29]$, is not supported in the present analyses of the family (Fig. 1). Our result agrees with the hypotheses of Seifried and Schminke [22] which proposed that, the genera Aegisthus, Andromastax, Jamstecia, Nudivorax and Scabrantenna Lee W. \& Huys, 2000 represent an advance possibly monophyletic group within Aegisthidae. The morphological characters that allowed the inclusion of the Aegisthinae together with former Cerviniinae and former Pontostratiotinae (sensu [30]) in the family Aegisthidae are the unique form of the anal somite (elongate and tapering posteriorly) and the modification of the posterior seta 11 (terminology following [21]) of the maxilla into a strong large spine [21, 22].
The unique spinous processes of the cephalothorax (arrow in Fig. 6b) and the extremely elongated furca (arrow in Fig. 6a) in Aegisthinae have been recognized by Seifried and Schminke [22] as derived characters shared with members of the former Pontostratiotinae (Pontostratiotes) (arrow in Fig. 6d,e) concluding that they belong to the same evolutionary lineage. However other harpacticoid reference literature (e.g. [29]) and even Seifried and Schminke [22] continued to consider former Pontostratiotinae and Aegisthinae to be valid, independent subfamilies. Our molecular analysis confirms the sister relationship between Aegisthinae and here re-defined Pontostratiotinae (which includes members of the Pontostratiotes only) and rejects the monophyly of the former Pontostratiotinae. 


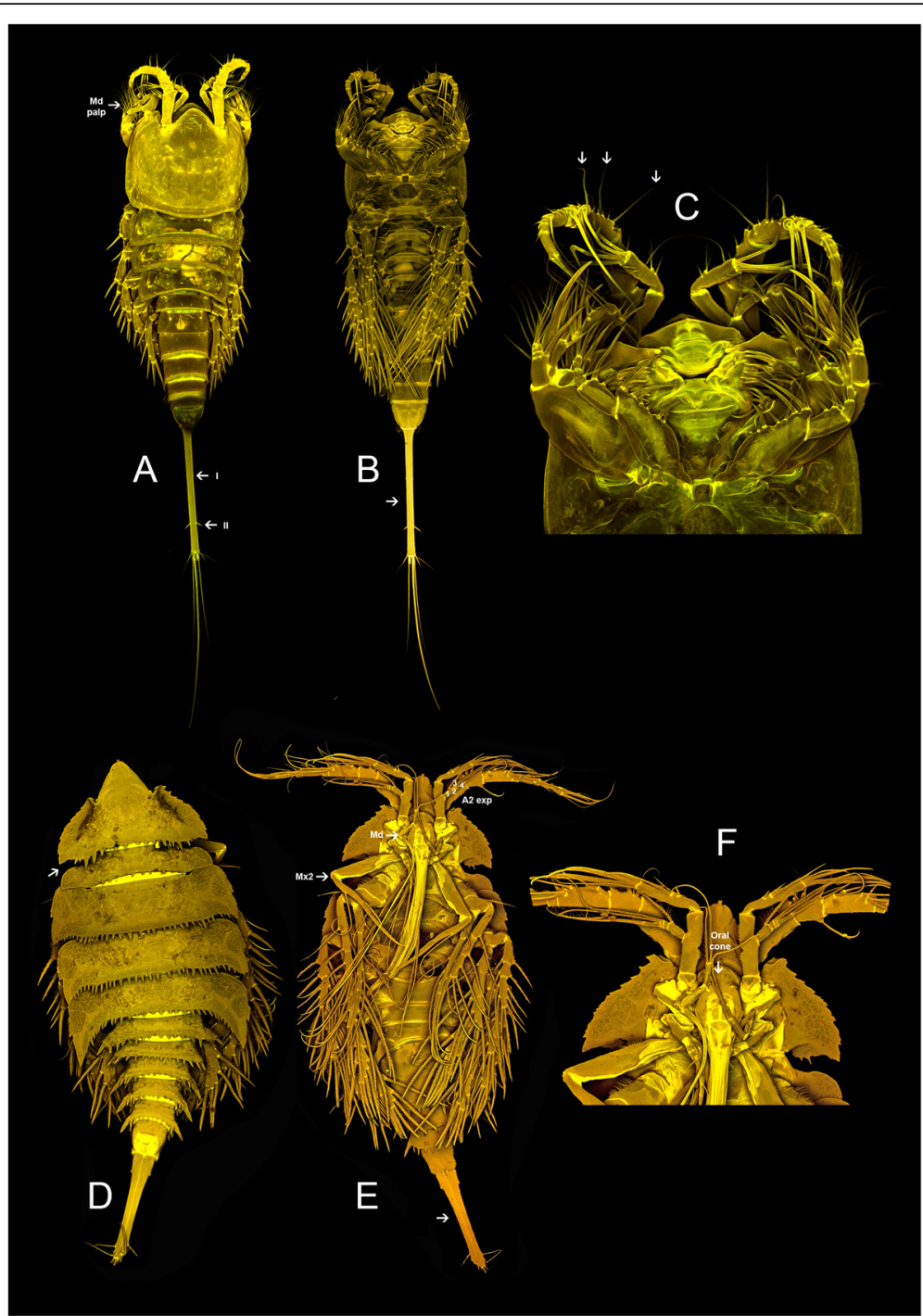

Fig. 4 Confocal laser scanning of representatives of the subfamily Cerviniopseinae. Stratiopontotes sp. habitus, dorsal (a), ventral (b) and mouthparts (c). Siphonis ruehlemanni. habitus, dorsal (d), ventral (e) and mouthparts (f)

In this study, the analyzed genera belonging to the Aegisthinae (Aegisthus, Andromastax, Nudivorax, and Jamstecia) have a complex topology (Fig. 1) in which there is no evidence supporting the monophyly of none of the four genera. Conroy Dalton and Huys [27] described Andromastax as the second known genus in the Aegisthidae, arguing that the inclusion of the new species within Aegisthus (the only genus recognized at that time) was impossible without grossly extending its generic boundaries. Some of the morphological characters used to define Andromastax were based on a combination of strong plesiomorphic character states (e.g. Md palp 2-segmented and bisetose; Mx1 basis with 8 elements; A2 exopod with 3 elements; P5 exopod with inner setae; P6 with 3 setae) and clearly defined apomorphies (e.g. dorsal spinous processes on cephalothorax, cephalosome with lateral spinous processes near bases of A2; lateral processes on coxae $\mathrm{P} 2-\mathrm{P} 4,+q$ second segment of A1 with 2 lateral processes on anterior margin, $\widehat{\sigma}$ Mx2 allobasis and enp-1 with modified pinnate spines [27]. We suggest that the dorsal spinous processes on the trunk somites and A1 (arrow in Fig. 6b, e) represent a synapomorphic character for the lineage formed by Aegisthinae + Pontostratiotinae which were lost in some members of Pontostratiotes and in the genus Nudivorax. Lee and Huys [19] described three monotypic genera of Aegisthinae from hydrothermal vents and cold seeps in Japan: Nudivorax, Scabrantenna and Jamstecia. Nudivorax is characterized by a complete lack of integumental surface reticulation and lack of spinous processes on both cephalosome and body somites which was considered to be unique among the Aegisthinae. Scabrantenna 


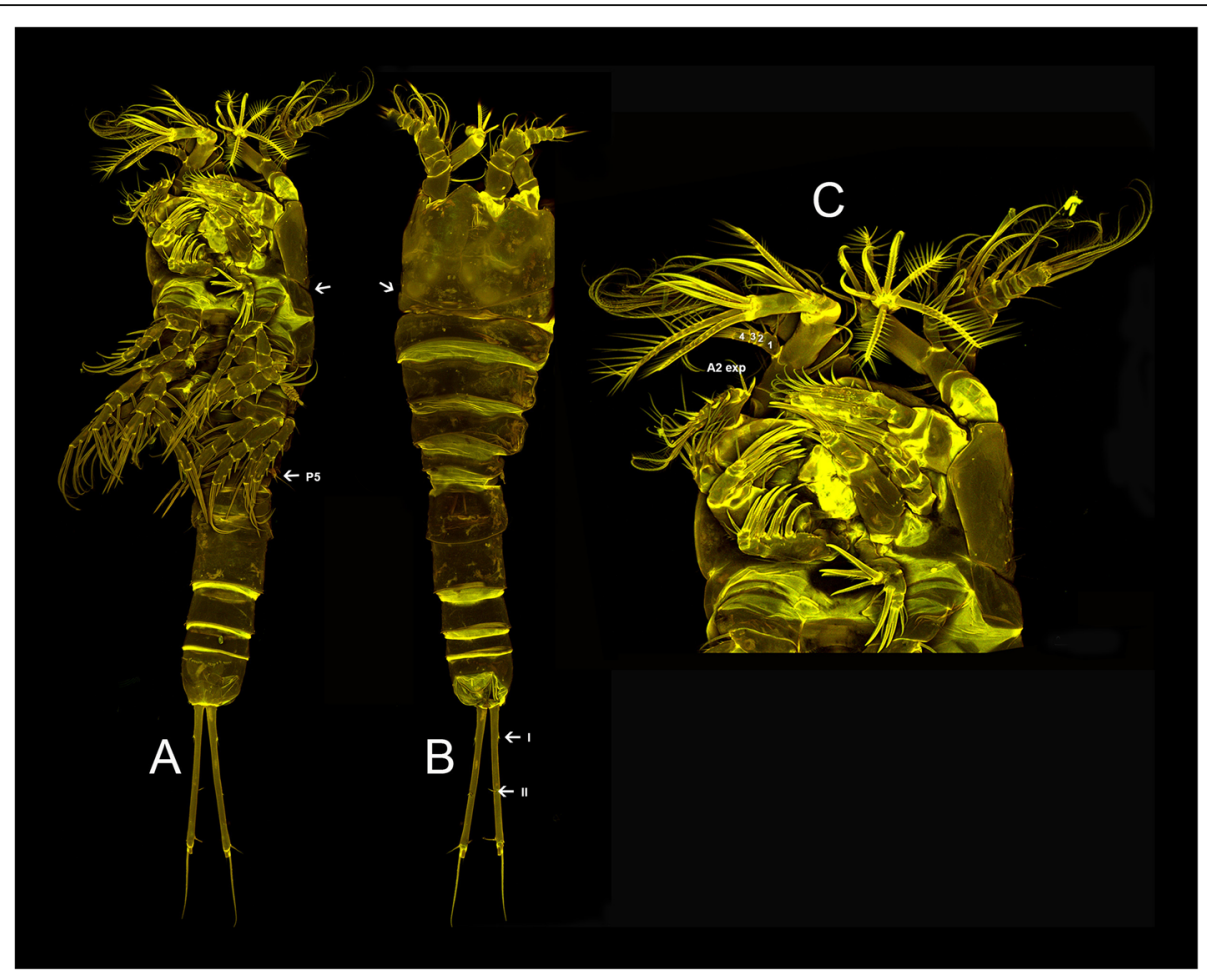

Fig. 5 Confocal laser scanning of the specimen representing subfamily Cerviniinae. Cervinia sp. habitus, dorsal (a), ventral (b) and mouthparts (c)

(missing from this analysis) is distinguished from other genera because of its sexually dimorphic $\mathrm{A} 1$ and its prehensile A2 (presumably used for mate guarding). The most distinctive character of Jamstecia is an elongated A1, resulting from secondary elongation of segment 1 (Fig. 6a, b), differing from other Aegisthinae in which the second segment is the longest. Lee and Huys [19] argued that Nudivorax could represent the most primitive genus within the family because of the plesiomorphic state of female A1 (which retains the maximum number of setae expressed in the family) and male mouthparts (retaining the full complement of armature as found in females) suggesting an early divergence within the subfamily. The above mentioned authors also discussed that Scabrantenna represents a transitional genus between the primitive Nudivorax and Andromastax compared with the advanced Aegisthus and questioned the position of the genus Jamstecia due to the lack of material for comparison. Lack of support in the monophyly of these four genera in our study demands providing DNA sequences from the type species of these genera for deeper molecular and morphological analysis. The materials we have sequenced correspond morphologically to the genera assigned in Fig. 1 but the species are not the name bearing types.

Here we, re-validate and re-define the subfamily Cerviniopseinae as a well-supported monophyletic group that includes the genera Stratiopontotes, Siphonis,
Cerviniopsis, Aegisthidae gen. 2., Hemicervinia, Herdmaniopsis and Tonpostratiotes. Stratiopontotes is here shown to be the most basal genus within the subfamily Cerviniopseinae. This genus mostly follows the ground pattern of the Aegisthidae in both cephalic and thoracic appendages, converging the molecular analysis with the morphology. Members of the genus Herdmaniopsis have been recognized as primitive forms within the former Pontostratiotinae by Lang [31], Brotskaya [9] and later by Ito [28]. The last author particularly emphasized the morphological similarities of the unarmed cephalic shield and thoracic pleuro-tergites of Stratiopontotes (Fig. 4a) and Herdmaniopsis, suggesting the close relationship between those genera. Herdmaniopsis has no representative in our molecular study, however we agree with Ito [28] and believe that Herdmaniopsis may be sister to Stratiopontotes in a basal position within Cerviniopseinae. Additionally, we agree with the clear separation of both genera because of the absence of setae on the first endopodite of the maxilliped and the shortened of the A1 in Herdmaniopsis, among other characters.

The only known associated genus of the family Aegisthidae, Siphonis, is shown here to be sister to Cerviniopsis. This genus is characterized by its siphonostomatoid-like mouthparts which include an elongated oral cone (formed by labrum and labium; that are sealed together by complex arrangement of overlapping ridges and grooves), a highly modified mandible (stylet-like) and maxillule and 


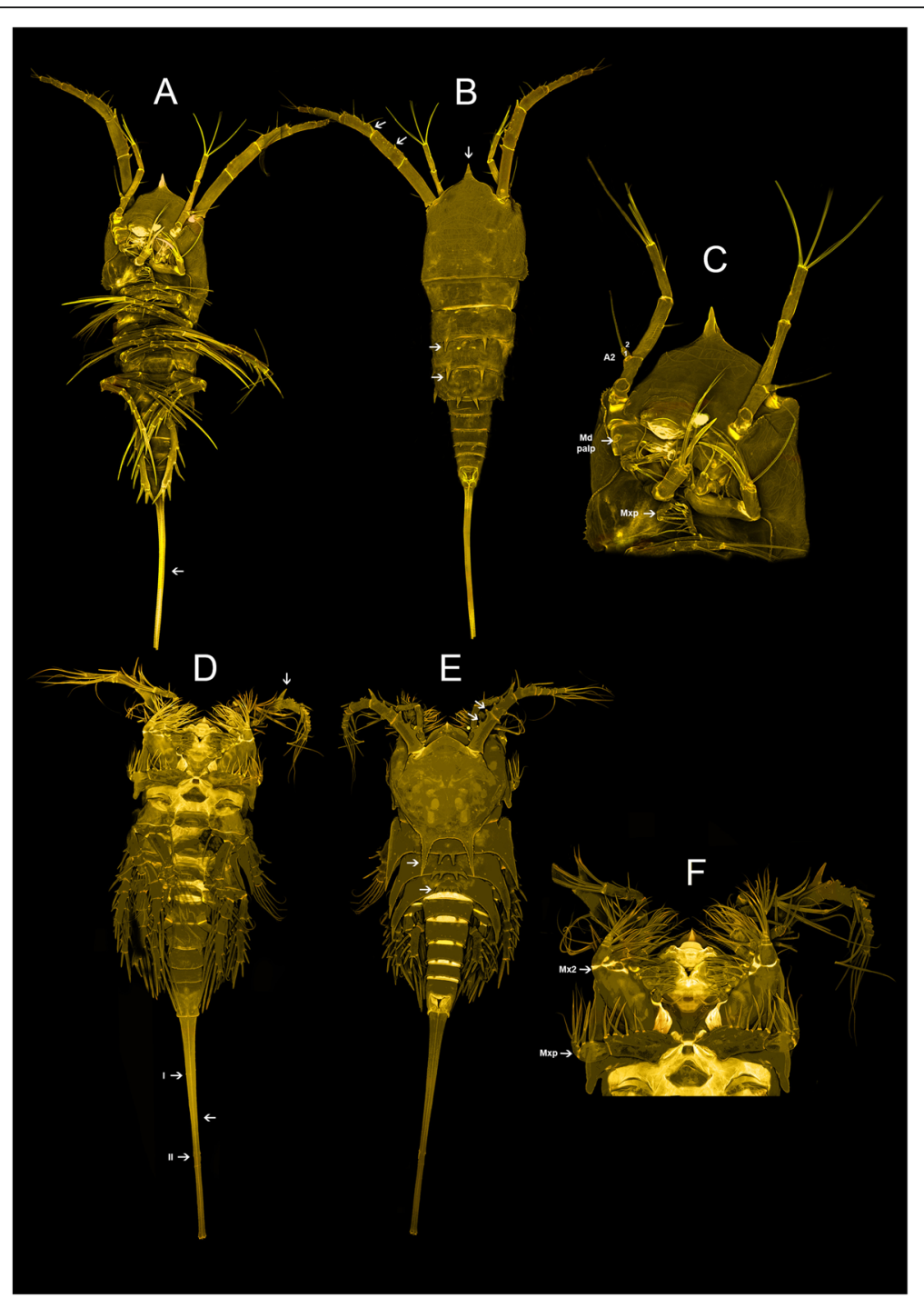

Fig. 6 Confocal laser scanning of the species representing subfamilies Pontostratiotinae and Aegisthinae. Jamstecia sp. habitus, dorsal (A), ventral (B) and mouthparts (C). Pontostratiotes sp. habitus, dorsal (D), ventral (E) and mouthparts (F)

maxilla with the same shape as in siphonostomatoids (arrow in Fig. 4e,f) [26]. Those analogous structures have previously been considered synapomorphies for the taxon Palinarthra Seifried, 2003 within the Harpacticoida [21]. The Palinarthra includes the families Novocriniidae Huys \& Iliffe, 1998, Superornatiremidae Huys, 1996, Rotundiclipeidae Huys, 1988, Peltidiidae Claus, 1860, Tegastidae Sars, G. O. 1904, and Porcellidiidae Boeck, 1865 as well as the families within the superfamily Tisboidea Stebbing, 1910 [21]. The short oral cone in Paninarthra families shows a similar morphology to the primitive families Asterocheridae Giesbrecht, 1899 and Dirivultidae Humes and Dojiri, 1980 of Siphonostomatoida [32, 33]; whereas, an elongated oral cone and highly modified mandible in Siphonis is considered a homoplasy with some particular Asterocheridae species such as Acontiophorus scutatus
(Brady and Robertson, 1873) and Scottocheres elongatus (Scott T. \& Scott A., 1894) both associated with Porifera. These similar modifications are characteristic of those associated copepods and have the functional task of penetrating and feeding from pores and channels in sponges (for a detailed discussion of the morphology and mouthpart modifications in the genus Siphonis see [26]).

Aegisthidae gen. 1 is, genetically, a well-supported independent lineage, sister to the subfamily Cerviniellinae subfam. nov. This undescribed genus was excluded from the Cerviniellinae subfam. nov. based on the distinct morphological differences between this genus compare to Hase and Cerviniella. The taxonomic status of the undescribed genus needs to be studied in details when additional material become available. Furthermore this genus is morphologically similar to the genus Stratiopontotes in the general armature 
and segmentation of A1, A2, mandible, maxillula, maxilla, maxilliped and P1-P5 (Fig. 2b, c). However it can be easily distinguished from Stratiopontotes because of its divergent furca (arrow in Fig. 2b), the robust and slightly projected labrum (arrow in Fig. 2c), the ratio of P1 endopod-exopod and; its general shape -being more robust and moderately dorso-ventrally flattened than Stratiopontotes- among other characters. Morphologically, the undescribed genus Aegisthidae gen. 1 agrees with the ground pattern of Harpacticoida in several important characters; one of them is the incomplete fusion of the first pedigerous somite from the dorsal cephalic shield (arrow in Fig. 2a shows dorsal suture). The complete fusion of the above mentioned somites has been considered as synapomorphy for the Syngnatharthra group which includes all families within Harpacticoida except for Aegisthidae and Rometidae [22]. However a complete secondary separation of the cephalosome and first pedigerous somite is presented in some species of Syngnatharthra as Chappuisiidae Chappuis, 1940, Phyllognathopodidae Gurney, 1932 and Latiremidae Bozic, 1969; or partially retained in members of Rotundiclipeidae and Tachidiidae Sars, G. O. 1909.

The subfamily Cerviniellinae subfam. nov. is here redefined to enclose members of the genus Cerviniella, Hase and Eucanuella, the latest is not included in the molecular analysis. Eucanuella is included within this subfamily because of the fusion of the first pedigerous somite to the cephalosome (similar to Cerviniella; arrow in Fig. 3e), the short A1 bearing several elements transformed into spines (arrow in Fig. 3f), the presence of strong hook-like lateral projections on the genital double somite (arrow in Fig. 3e, f) and the P1 exp. bended inwards (similar to Hase; arrow in Fig. 3a), the last three characters considered here as apomorphies to the Cerviniellinae subfam. Nov.

The genera Neocervinia and Pseudocervinia were synonymized with Cervinia, based on a 'strict consensus tree' [21], who considered the female one segmented P5 (arrow in Fig. 5a) as the only apomorphic character of Neocervinia. Later, Park et al. [20] endorsed the generic status of Neocervinia adding the presence of an aesthetasc (incorrectly referred by the authors as sensilla) on the A1 second and third segments as another synapomorphy for the genus (see [19, 34] for detailed characters). Our molecular analysis is incongruent with synonymizing Neocervinia with Cervinia, however re-validation of this taxon is postponed providing more material from this genus.

\section{Conclusions}

The family Aegisthidae has colonized a variety of habitats in the course of its diversification (Fig. 7). The deep-

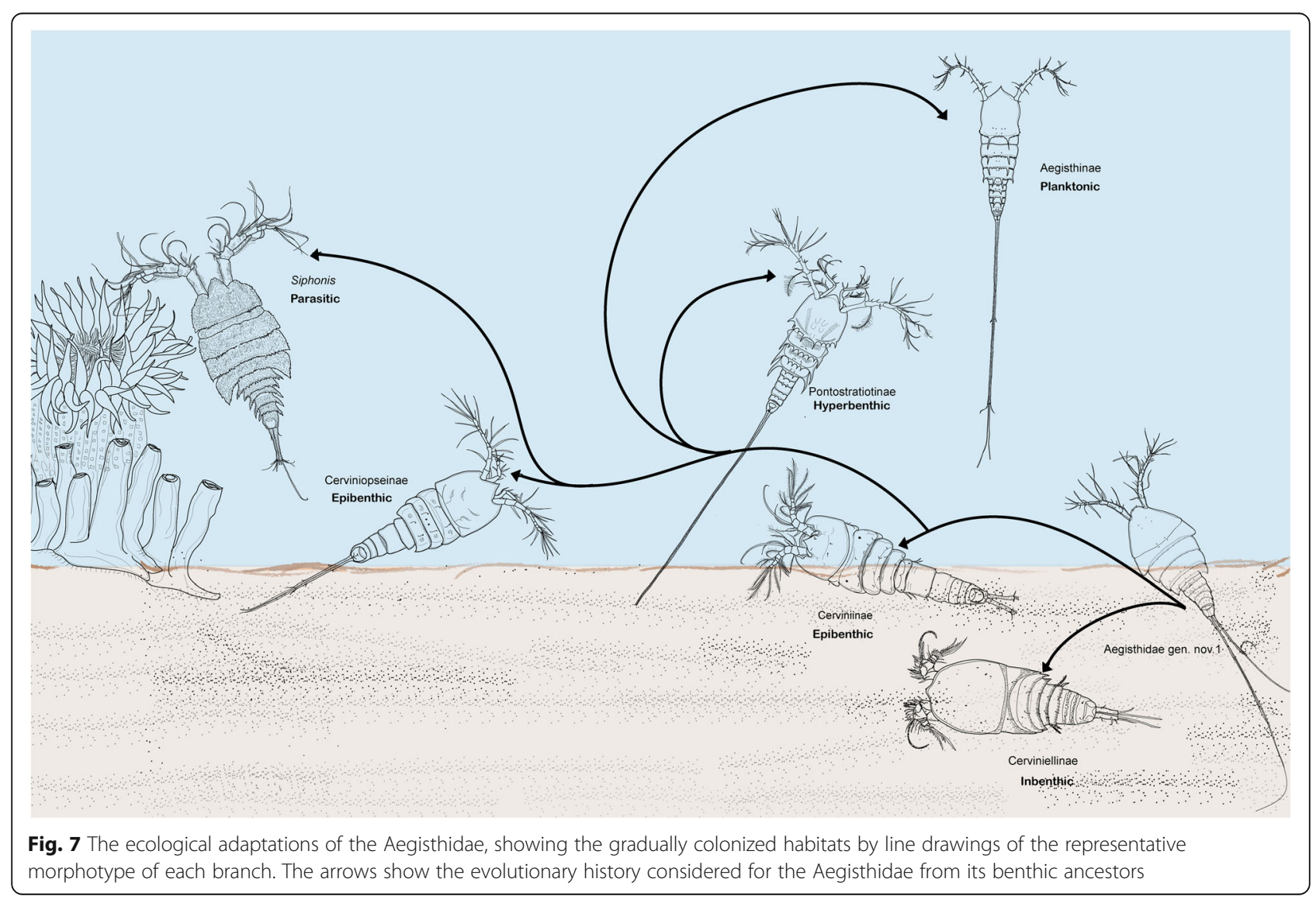


sea benthic origin of Aegisthidae [9,35] is demonstrated by the basal lineages such as Aegisthidae gen. 1 and Cerviniellinae subfam. nov. These species live on and in the sediments, but may also swim in the water close to the bottom (epibenthic). Broad spade-like appendages which are associated with burrowing lifestyle [13, 36, 37] are adaptations in benthic Cerviniellinae subfam. nov. species. These adaptations involve a stout and robust body shape, commonly with short furca, reduction in the length of the A1 segments (always less than one fifth of the total length of cephalosome), the development of robust modified setae in the A1, reductions in swimming legs (e.g. P1 endopod reduced to a single segment or absent in some species), swimming legs bent inwardly and with strongly developed spines and processes that may help in sediment removal.

Secondary adaptations to a pelagic existence in Aegisthidae $[25,38]$ are clearly distinguished in Pontostratiotinae in which extremely long furca, well developed spinous process and an increased body surface aid in avoidance of sinking are present. Members of the genus Pontostratiotes inhabit hyperbenthic water layers. The derived Aegisthinae, such as the planktonic species of the genus Aegisthus the increase in the length of the A1, A2 and furca and the segments of swimming legs are shown here to play an important role in the successful colonization of pelagic realm of the ocean $[8,9,13,25,31,38]$. The most advanced life style in this family is displayed by Siphonis, where mouth parts have been adapted to an associated life style, with a long siphonostomatoid-like oral cone, stylet-like mandible and siphostomatoid-like maxillula. Here, a comprehensive molecular phylogeny of the Aegisthidae shows that evolutionary trends in this family have begun from a benthic habit then to an epibenthic, hyperbenthic, planktonic then associated lifestyle.

\section{Methods}

\section{Taxon sampling}

The Aegisthidae species analyzed for this study were collected during four research cruises. The Atlantic Ocean were sampled around Iceland during the cruise ME 853 IceAGE onboard RV Meteor ([39] https://www.ldf.unihamburg.de/meteor/wochenberichte.html). Most specimens included in this study were obtained from three other cruises in the Pacific Ocean by RV SONNE, the first in the abyssal and hadal zones of the KurilKamchatka Trench SO250 ([40] https://doi.org/10.4126/ FRL01-006401131), the second in the Clarion Clipperton Fracture Zone, SO239 ([41] http://oceanrep.geomar.de/3 0422/) and the third, SO242-1 ecological aspect of deep-sea mining in the manganese nodule area of the south east Pacific ([42]; https://www.portal-forschungsschiffe.de/lw_resource/datapool/_items/item_14 8/so-242_1_fahrtbericht.pdf.)
Samples were preserved in 96\% ethanol and specimens were sorted using a dissecting microscope. Aegisthidae specimens were isolated and stored in 96\% ethanol at $20^{\circ} \mathrm{C}$. Species were identified to the lowest taxonomic level using diagnostic morphological characteristics. Many of the collected species are new to science and not yet described. Ninety four specimens were available representing the currently valid subfamilies of Aegisthidae: Aegisthinae (10 species) comprising 4 genera, Aegisthus, Andromastax, Jamstecia and Nudivorax; Cerviniinae (18 species) including 6 genera, Aegisthidae gen. 1, Cerviniella, Neocervinia, Cervinia, Expansicervinia and Hase; Pontostratiotinae (20 species) including 5 genera, Stratiopontotes, Siphonis, Cerviniopsis, Pontostratiotes and Aegisthidae gen. 2. Table 1 shows collected taxa and sampling coordinates.

\section{Molecular analysis}

DNA extractions were carried out using $40 \mu \mathrm{L}$ Chelex (InstaGene Matrix, Bio-Rad) following the protocol of Estoup et al. [43] from whole individuals and supernatant was stored at $-20^{\circ} \mathrm{C}$ for later DNA analysis. Exoskeletons of the extracted specimens were fixed in glycerin on a glass slide and stored as a voucher for morphological identifications. DNA was analyzed for all 94 specimens available for this study (Table 1). Phylogenetic analyses were carried out using $914 \mathrm{bp}$ of nuclear large (28S), $1792 \mathrm{bp}$ of small (18S) subunits of rRNA and $741 \mathrm{bp}$ of mitochondrial protein cytochrome $c$ oxidase subunit I (COI). Amplification was performed using AccuStart II GelTrack PCR SuperMix (ThermoFisher Scientific) or Phire Green Hot Start II PCR Master Mix (ThermoFisher Scientific) in a $25 \mu \mathrm{L}$ volume containing $9.5 \mu \mathrm{L} \mathrm{H} 2 \mathrm{O}, 12.5 \mu \mathrm{L}$ PCR Master Mix, $0.5 \mu \mathrm{L}$ of each primer $\left(10 \mathrm{pmol} \mu \mathrm{L}^{-1}\right)$ and $2 \mu \mathrm{L}$ of DNA template. PCR products were checked by electrophoresis on a $1 \%$ agarose/TAE gel containing $1 x$ GelRed. PCR products have been purified using EXO SAP (PCR cleaning, ThermoFisher Scientific) prior sequencing. Table 2 shows related PCR primers for each gene. Forward and reverse sequences were assembled and edited using Geneious (version 9.1.8 Biomatters; http://www.geneious.com). MAFFT v7.017 [50] was used to align trimmed DNA sequences for each gene under E-INS-i algorithm for nuclear genes and G-INS-i for COI [51]. The three alignments were concatenated and manually edited for ambiguous regions using Geneious v1.9.8. In order to root the tree, three species of Misophrioida Gurney, 1933 and two species of Canuelloida Khodami, Vaun MacArthur, Blanco-Bercial \& Martinez Arbizu, 2017 [52] were chosen as outgroups. The phylogenetic analyses were conducted using Bayesian inferences using MrBayes MPI version v3.2.2 ×64 [53, 54]. GTRGAMMA (General time reversible following gamma shape distribution) substitutional model were used for phylogenetic analyses as the best nucleotide fitting model for $18 \mathrm{~S}$ and $28 \mathrm{~S}$ 
Table 1 Taxon sampling of the Aegisthidae species collected and sequenced for this study. Sampling stations and coordinates are listed for each specimen

\begin{tabular}{|c|c|c|c|c|c|c|c|}
\hline ID & Taxon & Expedition & Location & Station & Latitude & Longitude & Depth (m) \\
\hline 331 & Aegisthidae gen.1 & SO239 & Clarion Clipperton Fracture Zone & 21 & $11^{\circ} 51,21^{\prime} \mathrm{N}$ & $117^{\circ} 3,57^{\prime} \mathrm{W}$ & 4146 \\
\hline K43 & Cerviniella brodskayae & SO250 & Kuril-Kamchatka Trench & 25 & $45^{\circ} 55,237^{\prime} \mathrm{N}$ & $152^{\circ} 47,467^{\prime} \mathrm{E}$ & 6066.4 \\
\hline K154 & Cerviniella brodskayae & SO250 & Kuril-Kamchatka Trench & 10 & $43^{\circ} 51,810^{\prime} \mathrm{N}$ & $151^{\circ} 46,543^{\prime} \mathrm{E}$ & 5188.1 \\
\hline K129 & Cerviniella sp. & SO250 & Kuril-Kamchatka Trench & 61 & $45^{\circ} 9997^{\prime} \mathrm{N}$ & $153^{\circ} 45,417^{\prime} \mathrm{E}$ & $5740,80,3$ \\
\hline K130 & Cerviniella sp. & SO250 & Kuril-Kamchatka Trench & 61 & $45^{\circ} 9997^{\prime} \mathrm{N}$ & $153^{\circ} 45,417^{\prime} \mathrm{E}$ & $5740,80,3$ \\
\hline 428 & Cerviniella sp. & SO239 & Clarion Clipperton Fracture Zone & 81 & $11^{\circ} 3,97^{\prime} \mathrm{N}$ & $119^{\circ} 37,67^{\prime} \mathrm{W}$ & 4365.7 \\
\hline K28 & Cerviniella lagarderei & SO250 & Kuril-Kamchatka Trench & 8 & $43^{\circ} 51,698^{\prime} N$ & $151^{\circ} 45,851^{\prime} \mathrm{E}$ & $5191,20,3$ \\
\hline K148 & Cerviniella sp. & SO250 & Kuril-Kamchatka Trench & 10 & $43^{\circ} 51,810^{\prime} \mathrm{N}$ & $151^{\circ} 46,543^{\prime} \mathrm{E}$ & 5188.1 \\
\hline K155 & Cerviniella sp. & SO250 & Kuril-Kamchatka Trench & 10 & $43^{\circ} 51,810^{\prime} \mathrm{N}$ & $151^{\circ} 46,543^{\prime} \mathrm{E}$ & 5188.1 \\
\hline K83 & Cerviniella sp. & SO250 & Kuril-Kamchatka Trench & 17 & $45^{\circ} 54,160^{\prime} \mathrm{N}$ & $153^{\circ} 54,685^{\prime}$ & 7994 \\
\hline K124 & Cerviniella sp. & SO250 & Kuril-Kamchatka Trench & 42 & $45^{\circ} 37,602^{\prime} N$ & $152^{\circ} 52,499^{\prime} \mathrm{E}$ & 6881.4 \\
\hline K134 & Cerviniella sp. & SO250 & Kuril-Kamchatka Trench & 62 & $45^{\circ} 9998^{\prime} \mathrm{N}$ & $153^{\circ} 45,418^{\prime} \mathrm{E}$ & 5742.5 \\
\hline K141 & Cerviniella sp. & SO250 & Kuril-Kamchatka Trench & 53 & $45^{\circ} 28,751^{\prime} \mathrm{N}$ & $153^{\circ} 11,649^{\prime} \mathrm{E}$ & 8941.4 \\
\hline 564 & Hase sp. & SO239 & Clarion Clipperton Fracture Zone & 124 & $13^{\circ} 51,28^{\prime} N$ & $123^{\circ} 14,69^{\prime} \mathrm{W}$ & 4510.8 \\
\hline D10 & Hase sp. & SO242-1 & Manganese Nodule area of the South East Pacific & 45 & $7^{\circ} 07,116^{\prime} \mathrm{S}$ & $88^{\circ} 26,356^{\prime} \mathrm{W}$ & 4184 \\
\hline 479 & Hase sp. & SO239 & Clarion Clipperton Fracture Zone & 50 & $11^{\circ} 49,92^{\prime} \mathrm{N}$ & $117^{\circ} 29,31^{\prime} \mathrm{W}$ & 4330.3 \\
\hline K152 & Hase sp. & SO250 & Kuril-Kamchatka Trench & 10 & $43^{\circ} 51,810^{\prime} \mathrm{N}$ & $151^{\circ} 46,543^{\prime} \mathrm{E}$ & 5188.1 \\
\hline K46 & Hase sp. & SO250 & Kuril-Kamchatka Trench & 10 & $43^{\circ} 51,810^{\prime} \mathrm{N}$ & $151^{\circ} 46,543^{\prime} \mathrm{E}$ & 5188.1 \\
\hline K44 & Hase sp. & SO250 & Kuril-Kamchatka Trench & 8 & $43^{\circ} 51,698^{\prime} N$ & $151^{\circ} 45,851^{\prime} \mathrm{E}$ & $5191,20,3$ \\
\hline 501 & Neocervinia sp. & SO239 & Clarion Clipperton Fracture Zone & 99 & $11^{\circ} 2,28^{\prime} \mathrm{N}$ & $119^{\circ} 40,89^{\prime} \mathrm{W}$ & 4401.4 \\
\hline 358 & Expansicervinia sp. & SO239 & Clarion Clipperton Fracture Zone & 20 & $11^{\circ} 50,31^{\prime} \mathrm{N}$ & $116^{\circ} 58,78^{\prime} \mathrm{W}$ & 4093 \\
\hline 558 & Expansicervinia sp. & SO239 & Clarion Clipperton Fracture Zone & 94 & $11^{\circ} 4,42^{\prime} \mathrm{N}$ & $119^{\circ} 39,33^{\prime} \mathrm{W}$ & 4414.4 \\
\hline 432 & Expansicervinia sp. & SO239 & Clarion Clipperton Fracture Zone & 81 & $11^{\circ} 4,29^{\prime} \mathrm{N}$ & $119^{\circ} 36,29^{\prime} \mathrm{W}$ & 4346.4 \\
\hline D31 & Expansicervinia sp. & SO242-1 & Manganese Nodule area of the South East Pacific & 37 & $7^{\circ} 07,854^{\prime} \mathrm{S}$ & $88^{\circ} 25,484^{\prime} \mathrm{W}$ & 4176.3 \\
\hline 634 & Expansicervinia sp. & SO239 & Clarion Clipperton Fracture Zone & 117 & $13^{\circ} 52,78^{\prime} \mathrm{N}$ & $123^{\circ} 13,82^{\prime} \mathrm{W}$ & 4513.1 \\
\hline K80 & Cervinia sp. & SO250 & Kuril-Kamchatka Trench & 17 & $45^{\circ} 54,160^{\prime} \mathrm{N}$ & $153^{\circ} 54,685^{\prime}$ & 7994 \\
\hline K149 & Cervinia sp. & SO250 & Kuril-Kamchatka Trench & 52 & $45^{\circ} 31,996^{\prime} \mathrm{N}$ & $153^{\circ} 15,993^{\prime} \mathrm{E}$ & 8358.4 \\
\hline 601 & Cervinia sp. & SO239 & Clarion Clipperton Fracture Zone & 158 & $14^{\circ} 3,41^{\prime} \mathrm{N}$ & $130^{\circ} 7,99^{\prime} \mathrm{W}$ & 4946 \\
\hline 575 & Cervinia sp. & SO239 & Clarion Clipperton Fracture Zone & 118 & $13^{\circ} 52,38^{\prime} N$ & $123^{\circ} 15,09^{\prime} \mathrm{W}$ & 4511.7 \\
\hline K123 & Cervinia sp. & SO250 & Kuril-Kamchatka Trench & 42 & $45^{\circ} 37,602^{\prime} \mathrm{N}$ & $152^{\circ} 52,499^{\prime} \mathrm{E}$ & 6881.4 \\
\hline K109 & Cervinia sp. & SO250 & Kuril-Kamchatka Trench & 40 & $45^{\circ} 39,976^{\prime} \mathrm{N}$ & $152^{\circ} 55,953^{\prime} \mathrm{E}$ & 7300.3 \\
\hline D22 & Expansicervinia sp. & SO242-1 & Manganese Nodule area of the South East Pacific & 93 & $6^{\circ} 59,902^{\prime} \mathrm{S}$ & $88^{\circ} 30 ; 764^{\prime} \mathrm{W}$ & 4142.1 \\
\hline K158 & Expansicervinia sp. & SO250 & Kuril-Kamchatka Trench & 10 & $43^{\circ} 51,810^{\prime} \mathrm{N}$ & $151^{\circ} 46,543^{\prime} \mathrm{E}$ & 5188.1 \\
\hline D30 & Expansicervinia sp. & SO242-1 & Manganese Nodule area of the South East Pacific & 37 & $7^{\circ} 07,854 \mathrm{~S}$ & $88^{\circ} 25,484^{\prime} \mathrm{W}$ & 4176.3 \\
\hline K33 & Stratiopontotes sp. & SO250 & Kuril-Kamchatka Trench & 8 & $43^{\circ} 51,698^{\prime} \mathrm{N}$ & $151^{\circ} 45,851^{\prime} \mathrm{E}$ & $5191,20,3$ \\
\hline K137 & Stratiopontotes sp. & SO250 & Kuril-Kamchatka Trench & 62 & $45^{\circ} 9998^{\prime} \mathrm{N}$ & $153^{\circ} 45,418^{\prime} \mathrm{E}$ & 5742.5 \\
\hline K132 & Stratiopontotes sp. & SO250 & Kuril-Kamchatka Trench & 61 & $45^{\circ} 9997^{\prime} \mathrm{N}$ & $153^{\circ} 45,417^{\prime} \mathrm{E}$ & $5740,80,3$ \\
\hline 523 & Siphonis ruehlemanni & SO239 & Clarion Clipperton Fracture Zone & 20 & $11^{\circ} 50,31^{\prime} \mathrm{N}$ & $116^{\circ} 58,78^{\prime} W$ & 4093 \\
\hline 298 & Siphonis ruehlemanni & SO239 & Clarion Clipperton Fracture Zone & 20 & $11^{\circ} 50,31^{\prime} \mathrm{N}$ & $116^{\circ} 58,78^{\prime} W$ & 4093 \\
\hline 543 & Siphonis aureus & SO239 & Clarion Clipperton Fracture Zone & 81 & $11^{\circ} 4,29^{\prime} \mathrm{N}$ & $119^{\circ} 36,29^{\prime} \mathrm{W}$ & 4346.4 \\
\hline 521 & Cerviniopsis sp. & SO239 & Clarion Clipperton Fracture Zone & 20 & $11^{\circ} 50,31^{\prime} \mathrm{N}$ & $116^{\circ} 58,78^{\prime} \mathrm{W}$ & 4093 \\
\hline 396 & Cerviniopsis longicaudata & SO239 & Clarion Clipperton Fracture Zone & 59 & $11^{\circ} 48,22^{\prime} \mathrm{N}$ & $117^{\circ} 30,42^{\prime} \mathrm{W}$ & 4324.5 \\
\hline 507 & Cerviniopsis sp. & SO239 & Clarion Clipperton Fracture Zone & 99 & $11^{\circ} 2,28^{\prime} \mathrm{N}$ & $119^{\circ} 40,89^{\prime} \mathrm{W}$ & 4401.4 \\
\hline
\end{tabular}


Table 1 Taxon sampling of the Aegisthidae species collected and sequenced for this study. Sampling stations and coordinates are listed for each specimen (Continued)

\begin{tabular}{|c|c|c|c|c|c|c|c|}
\hline ID & Taxon & Expedition & Location & Station & Latitude & Longitude & Depth $(\mathrm{m}$ \\
\hline 562 & Cerviniopsis sp. & SO239 & Clarion Clipperton Fracture Zone & 99 & $11^{\circ} 2,28^{\prime} \mathrm{N}$ & $119^{\circ} 40,89^{\prime} \mathrm{W}$ & 4401.4 \\
\hline 510 & Cerviniopsis sp. & SO239 & Clarion Clipperton Fracture Zone & 99 & $11^{\circ} 2,28^{\prime} \mathrm{N}$ & $119^{\circ} 40,89^{\prime} \mathrm{W}$ & 4401.4 \\
\hline K196 & Cerviniopsis sp. & SO250 & Kuril-Kamchatka Trench & 86 & $45^{\circ} 1202^{\prime} \mathrm{N}$ & $151^{\circ} 6008^{\prime} \mathrm{E}$ & 5571.6 \\
\hline 505 & Cerviniopsis sp. & SO239 & Clarion Clipperton Fracture Zone & 99 & $11^{\circ} 2,28^{\prime} \mathrm{N}$ & $119^{\circ} 40,89^{\prime} \mathrm{W}$ & 4401.4 \\
\hline K36 & Aegisthidae gen.2 & SO250 & Kuril-Kamchatka Trench & 8 & $43^{\circ} 51,698^{\prime} \mathrm{N}$ & $151^{\circ} 45,851^{\prime} \mathrm{E}$ & $5191,20,3$ \\
\hline K163 & Nudivorax sp. & SO250 & Kuril-Kamchatka Trench & 55 & $45^{\circ} 29,242^{\prime} \mathrm{N}$ & $153^{\circ} 13,453^{\prime} \mathrm{E}$ & 8734.4 \\
\hline K125 & Jamstecia sp. & SO250 & Kuril-Kamchatka Trench & 5 & $43^{\circ} 49,196^{\prime} \mathrm{N}$ & $151^{\circ} 45,593^{\prime} \mathrm{E}$ & 5149.4 \\
\hline D18 & Jamstecia sp. & SO242-1 & Manganese Nodule area of the South East Pacific & 81 & $7^{\circ} 03,442 S$ & $88^{\circ} 28,903^{\prime} \mathrm{W}$ & 4152.9 \\
\hline 301 & Andromastax sp. & SO239 & Clarion Clipperton Fracture Zone & 20 & $11^{\circ} 50,31^{\prime} \mathrm{N}$ & $116^{\circ} 58,78^{\prime} \mathrm{W}$ & 4093 \\
\hline 411 & Andromastax sp. & SO239 & Clarion Clipperton Fracture Zone & 50 & $11^{\circ} 49,92^{\prime} \mathrm{N}$ & $117^{\circ} 29,31^{\prime} \mathrm{W}$ & 4330.3 \\
\hline 365 & Andromastax sp. & SO239 & Clarion Clipperton Fracture Zone & 20 & $11^{\circ} 50,31^{\prime} \mathrm{N}$ & $116^{\circ} 58,78^{\prime} \mathrm{W}$ & 4093 \\
\hline 367 & Andromastax sp.Andr & SO239 & Clarion Clipperton Fracture Zone & 20 & $11^{\circ} 50,31^{\prime} \mathrm{N}$ & $116^{\circ} 58,78^{\prime} \mathrm{W}$ & 4093 \\
\hline K34 & Nudivorax sp. & SO250 & Kuril-Kamchatka Trench & 8 & $43^{\circ} 51,698^{\prime} \mathrm{N}$ & $151^{\circ} 45,851^{\prime} \mathrm{E}$ & $5191,20,3$ \\
\hline 368 & Aegisthus sp. & SO239 & Clarion Clipperton Fracture Zone & 45 & $7^{\circ} 07,116^{\prime} \mathrm{S}$ & $88^{\circ} 26,356^{\prime} \mathrm{W}$ & 4184 \\
\hline K159 & Jamstecia sp. & SO250 & Kuril-Kamchatka Trench & 10 & $43^{\circ} 51,810^{\prime} \mathrm{N}$ & $151^{\circ} 46,543^{\prime} \mathrm{E}$ & 5188.1 \\
\hline 373 & Aegisthus sp. & SO239 & Clarion Clipperton Fracture Zone & 20 & $11^{\circ} 50,31^{\prime} \mathrm{N}$ & $116^{\circ} 58,78^{\prime} \mathrm{W}$ & 4093 \\
\hline 371 & Aegisthus sp. & SO239 & Clarion Clipperton Fracture Zone & 20 & $11^{\circ} 50,31^{\prime} \mathrm{N}$ & $116^{\circ} 58,78^{\prime} \mathrm{W}$ & 4093 \\
\hline D34 & Aegisthus sp. & SO242-1 & Manganese Nodule area of the South East Pacific & 37 & $7^{\circ} 07,854 \mathrm{~S}$ & $88^{\circ} 25,484^{\prime} \mathrm{W}$ & 4176.3 \\
\hline 619 & Andromastax sp. & SO239 & Clarion Clipperton Fracture Zone & 118 & $13^{\circ} 52,38^{\prime} \mathrm{N}$ & $123^{\circ} 15,09^{\prime} \mathrm{W}$ & 4511.7 \\
\hline 616 & Andromastax sp. & SO239 & Clarion Clipperton Fracture Zone & 171 & $14^{\circ} 2,68^{\prime} \mathrm{N}$ & $130^{\circ} 5,97^{\prime} \mathrm{W}$ & 5030.2 \\
\hline 576 & Andromastax sp. & SO239 & Clarion Clipperton Fracture Zone & 118 & $13^{\circ} 52,38^{\prime} \mathrm{N}$ & $123^{\circ} 15,09^{\prime} \mathrm{W}$ & 4511.7 \\
\hline K128 & Pontostratiotes sp. & SO250 & Kuril-Kamchatka Trench & 23 & $45^{\circ} 57,724^{\prime} \mathrm{N}$ & $152^{\circ} 39,836^{\prime} \mathrm{E}$ & 5959.2 \\
\hline K127 & Pontostratiotes sp. & SO250 & Kuril-Kamchatka Trench & 23 & $45^{\circ} 57,724^{\prime} \mathrm{N}$ & $152^{\circ} 39,836^{\prime} E$ & 5959.2 \\
\hline 304 & Pontostratiotes sp. & SO239 & Clarion Clipperton Fracture Zone & 20 & $11^{\circ} 50,31^{\prime} \mathrm{N}$ & $116^{\circ} 58,78^{\prime} \mathrm{W}$ & 4093 \\
\hline 303 & Pontostratiotes sp. & SO239 & Clarion Clipperton Fracture Zone & 20 & $11^{\circ} 50,31^{\prime} \mathrm{N}$ & $116^{\circ} 58,78^{\prime} \mathrm{W}$ & 4093 \\
\hline 499 & Pontostratiotes sp. & SO239 & Clarion Clipperton Fracture Zone & 99 & $11^{\circ} 2,28^{\prime} \mathrm{N}$ & $119^{\circ} 40,89^{\prime} \mathrm{W}$ & 4401.4 \\
\hline D11 & Pontostratiotes sp. & SO242-1 & Manganese Nodule area of the South East Pacific & 37 & $7^{\circ} 07,854 \mathrm{~S}$ & $88^{\circ} 25,484^{\prime} \mathrm{W}$ & 4176.3 \\
\hline 30 & Pontostratiotes fontani & ME 85-3 & North Atlantic around Iceland & 1054 & $61^{\circ} 57,33 \mathrm{~N}$ & $31^{\circ} 38,583^{\prime} \mathrm{W}$ & 2548 \\
\hline 31 & Pontostratiotes fontani & ME 85-3 & North Atlantic around Iceland & 1054 & $61^{\circ} 57,33 \mathrm{~N}$ & $31^{\circ} 38,583^{\prime} \mathrm{W}$ & 2548 \\
\hline 521 & Pontostratiotes sp. & SO239 & Clarion Clipperton Fracture Zone & 20 & $11^{\circ} 50,31^{\prime} \mathrm{N}$ & $116^{\circ} 58,78^{\prime} \mathrm{W}$ & 4093 \\
\hline D46 & Pontostratiotes sp. & SO242-1 & Manganese Nodule area of the South East Pacific & 45 & $7^{\circ} 07,116^{\prime} \mathrm{S}$ & $88^{\circ} 26,356^{\prime} W$ & 4184 \\
\hline 520 & Pontostratiotes sp. & SO239 & Clarion Clipperton Fracture Zone & 20 & $11^{\circ} 50,31^{\prime} \mathrm{N}$ & $116^{\circ} 58,78^{\prime} \mathrm{W}$ & 4093 \\
\hline 518 & Pontostratiotes sp. & SO239 & Clarion Clipperton Fracture Zone & 20 & $11^{\circ} 50,31^{\prime} \mathrm{N}$ & $116^{\circ} 58,78^{\prime} \mathrm{W}$ & 4093 \\
\hline 300 & Pontostratiotes sp. & SO239 & Clarion Clipperton Fracture Zone & 20 & $11^{\circ} 50,31^{\prime} \mathrm{N}$ & $116^{\circ} 58,78^{\prime} \mathrm{W}$ & 4093 \\
\hline D24 & Pontostratiotes sp. & SO242-1 & Manganese Nodule area of the South East Pacific & 81 & $7^{\circ} 03,442 S$ & $88^{\circ} 28,903^{\prime} \mathrm{W}$ & 4152.9 \\
\hline D21 & Pontostratiotes sp. & SO242-1 & Manganese Nodule area of the South East Pacific & 81 & $7^{\circ} 03,442 S$ & $88^{\circ} 28,903^{\prime} \mathrm{W}$ & 4152.9 \\
\hline K30 & Pontostratiotes sp. & SO250 & Kuril-Kamchatka Trench & 8 & $43^{\circ} 51,698^{\prime} N$ & $151^{\circ} 45,851^{\prime} \mathrm{E}$ & $5191,20,3$ \\
\hline 299 & Pontostratiotes sp. & SO239 & Clarion Clipperton Fracture Zone & 20 & $11^{\circ} 50,31^{\prime} \mathrm{N}$ & $116^{\circ} 58,78^{\prime} \mathrm{W}$ & 4093 \\
\hline K93 & Pontostratiotes sp. & SO250 & Kuril-Kamchatka Trench & 30 & $45^{\circ} 56,838^{\prime} \mathrm{N}$ & $152^{\circ} 50,939^{\prime} \mathrm{E}$ & 6165.1 \\
\hline K31 & Pontostratiotes gladius & SO250 & Kuril-Kamchatka Trench & 8 & $43^{\circ} 51,698^{\prime} N$ & $151^{\circ} 45,851^{\prime} \mathrm{E}$ & $5191,20,3$ \\
\hline 517 & Pontostratiotes sp. & SO239 & Clarion Clipperton Fracture Zone & 20 & $11^{\circ} 50,31^{\prime} \mathrm{N}$ & $116^{\circ} 58,78^{\prime} \mathrm{W}$ & 4093 \\
\hline D15 & Pontostratiotes sp. & SO242-1 & Manganese Nodule area of the South East Pacific & 81 & $7^{\circ} 03,4425$ & $88^{\circ} 28,903^{\prime} \mathrm{W}$ & 4152.9 \\
\hline D47 & Pontostratiotes sp. & SO242-1 & Manganese Nodule area of the South East Pacific & 93 & $6^{\circ} 59,902^{\prime} \mathrm{S}$ & $88^{\circ} 30 ; 764^{\prime} W$ & 4142.1 \\
\hline
\end{tabular}


Table 1 Taxon sampling of the Aegisthidae species collected and sequenced for this study. Sampling stations and coordinates are listed for each specimen (Continued)

\begin{tabular}{|c|c|c|c|c|c|c|c|}
\hline ID & Taxon & Expedition & Location & Station & Latitude & Longitude & Depth $(\mathrm{m})$ \\
\hline $\bar{D} 25$ & Pontostratiotes sp. & SO242-1 & Manganese Nodule area of the South East Pacific & 81 & $7^{\circ} 03,442 S$ & $88^{\circ} 28,903^{\prime} \mathrm{W}$ & 4152.9 \\
\hline D37 & Pontostratiotes sp. & SO242-1 & Manganese Nodule area of the South East Pacific & 45 & $7^{\circ} 07,116^{\prime} \mathrm{S}$ & $88^{\circ} 26,356^{\prime} \mathrm{W}$ & 4184 \\
\hline 519 & Pontostratiotes sp. & SO239 & Clarion Clipperton Fracture Zone & 20 & $11^{\circ} 50,31^{\prime} \mathrm{N}$ & $116^{\circ} 58,78^{\prime} \mathrm{W}$ & 4093 \\
\hline 320 & Pontostratiotes sp. & SO239 & Clarion Clipperton Fracture Zone & 24 & $11^{\circ} 51,87^{\prime} \mathrm{N}$ & $116^{\circ} 59,74^{\prime} \mathrm{W}$ & 4122 \\
\hline D23 & Pontostratiotes sp. & SO242-1 & Manganese Nodule area of the South East Pacific & 45 & $7^{\circ} 07,116^{\prime} \mathrm{S}$ & $88^{\circ} 26,356^{\prime} W$ & 4184 \\
\hline D35 & Pontostratiotes sp. & SO242-1 & Manganese Nodule area of the South East Pacific & 45 & $7^{\circ} 07,116^{\prime} \mathrm{S}$ & $88^{\circ} 26,356^{\prime} W$ & 4184 \\
\hline K29 & Pontostratiotes sp. & SO250 & Kuril-Kamchatka Trench & 8 & $43^{\circ} 51,698^{\prime} \mathrm{N}$ & $151^{\circ} 45,851^{\prime} \mathrm{E}$ & $5191,20,3$ \\
\hline 313 & Pontostratiotes sp. & SO239 & Clarion Clipperton Fracture Zone & 20 & $11^{\circ} 50,31^{\prime} \mathrm{N}$ & $116^{\circ} 58,78^{\prime} \mathrm{W}$ & 4093 \\
\hline
\end{tabular}

rRNA calculated by jModeltest v0.1.1 [55] under Java. The codon model was implemented for the COI partition following the nexus block: lset Nucmodel = codon, code $=$ metmt, omegavar $=$ M3, nst $=$ mix and rates $=$ gamma. Two different MrBayes character partitions were provided and compared in two independent MrBayes jobs for the phylogenetic trees: job 1) considering $18 \mathrm{~S}$ and $28 \mathrm{~S}$ in one partition under $n s t=6($ GTR) rate = gamma; job 2) allocating individual partition for $18 \mathrm{~S}$ and $28 \mathrm{~S}$ in which the nst $=6$ and rate = gamma were applied to each partition separately. Posterior probabilities were estimated using 8,792, 000 generations through four simultaneous Markov Chains Monte Carlo for the number of 2 runs and sample frequency of every 1000 trees. The average standard deviation of split frequencies between 2 runs reached the value of 0.007 for job 1 and 0.004 for job 2. The ESS (estimated sampling size) is more than 300 for all estimated parameters in job1 in which the ESS value of the LnL has been estimated to 1408-1561 for both runs. However in Job 2 the ESS value of some parameters are less than minimum accepted (Supplementary information) which indicates that the posterior was not effectively sampled during this single run. A $50 \%$ majority rule consensus tree with median

Table 2 PCR and sequencing primers used for this study

\begin{tabular}{lll}
\hline Primer & Fragment & $\begin{array}{l}\text { Annealing } \\
\text { Temperature }\end{array}$ \\
\hline 18SE-F [44] & 18S rRNA & $51{ }^{\circ} \mathrm{C}$ \\
18SL-R [45] & \\
F1 [46] & \\
CF2 [46] & \\
CR1 [46] & \\
R2 [46] & \\
28S-F1a [47] & \\
28S-R1a [47] & \\
LCO 1490 [48] & \\
HCO 2198 [48] & & \\
Cop-COI-2189R [49] & COI mt DNA & \\
\hline
\end{tabular}

branch lengths was made, discarding the $25 \%$ of the first trees (relburnin = yes) from each job individually. All generated trees (.t files) and estimated priors (.p files) are available as supplementary information for both MrBayes jobs (Additional files 2 and 3). The gene fragments sequenced in this study are available in Genbank following the accession numbers MN536817 - MN536902 for 18S rRNA, MN536171 - MN536215 for COI mtDNA and MN535552 - MN535623 for 28S rRNA.

\section{Systematic part}

Definition of the subfamily Cerviniellinae subfam. nov., re-definition of the subfamilies Cerviniinae, Cerviniopseinae, Pontostratiotinae and Aegisthinae have been provided bellow. All abbreviation used in this section has been lifted in section 7 (List of abbreviations). Caudal setae labeled as follows: I- anterolateral accessory seta; II- anterolateral (lateral) caudal seta; III- posterolateral (outermost) caudal seta; IV-outer terminal (terminal median external) caudal seta; V-inner terminal (terminal median internal) caudal seta; VI-terminal accessory (innermost) caudal seta; VII- dorsal seta; nomenclature follows Huys and Boxshall [56]. The terms furca and telson are used following Schminke [57].

\section{Groundpattern of Aegisthidae (after [21])}

Female. Prosome and urosome clearly separated, prosome consisting of cephalosome and 4 free pedigerous somites; first pedigerous somite separated from dorsal cephalic shield. Urosome 5-segmented comprising of fifth pedigerous somite, genital double-somite and three free abdominal somites. Anal somite elongate, tapering posteriorly. Furca more than twice as long as wide, bearing 7 furca setae. Antennule (A1) 8-segmented; armature formula: 1(1s), 2(12s), 3(14s + 1ae), 4(2s), 5(3s), 6(2s), $7(2 s), 8(6 s+1$ aecrothek $)$. Oligoarthra segments $3+4$ fused. Antenna (A2) with coxa, incomplete allobasis and 1 free endopodal segment; basis and endopod 1 (enp1) fused, bearing 1 seta each; enp 2 with 1 spine and 2 setae on lateral margin and 7 distal setae; Exopod (exp) 4- 
segmented, armature formula: 1(2 s), 2(1 s), 3(1 s), 4(3 s). Mandible (Md) with coxa bearing a well-develop gnathobase; palp formed by basis, enp and exp.; basis with 4 setae; enp large (at least 2 times longer than wide), with 1 spine and 2 setae laterally and 7 distal setae, spine I lacking; exp. 4-segmented with 2,1,1,2 setae. (Maxillula) Mx1 comprising praecoxa, coxa, exp. and basis fused with enp; epipodite represented by 2 setae; fused basis and enp with 14 setae; exp. reduced in size bearing 3 setae. (Maxilla) Mx2 4-segmented comprising syncoxa, allobasis and three segmented enp; endopodal element 11 of allobasis developed as large, strong spine inserted on posterior surface. (Maxilliped) Mxp 4-segmented, comprising syncoxa, basis and 2-segmented enp; coxa with incorporated endites; basis with 1 spine and 1 seta; enp with $3 \mathrm{~s}, 2 \mathrm{sp}+2 \mathrm{~s}$. (Swimming legs 1 to 4) P1-P4 with 3-segmented rami. P5 without endopodal lobe; exp. more than twice as long as wide, armature: 1 inner spine, 3 outer spines, and 1 setae and 2 spines around apex. P6 with 3 setae.

Male: Sexual dimorphism in body size, genital segmentation, A1, P5 and P6. Caudal setae I and II transformed into strong spines. Urosome (Urs) 6-segmented, comprising of fifth pedigerous somite and 4 abdominal somites, 2 spermatophores. A1 10-segmented; armature: 1(1s), 2(11s + 1ae), 3(6s+1ae), 4(2s), 5(3s+1ae), 6(2s), $7(2 s), 8(3 s), 9(4 s), 10(10 s+1$ aecrothek); fusion of Oligoarthra segments 2 and 3, 10 and 11, 12 to 14 . P5 without endopodal lobe, basis separated from coxa, and 3segmented exp. P6 with 3 setae.

\section{Cerviniellinae subfam. nov.}

\section{Type genus: Cerviniella Smirnov, 1946}

Other genera; Eucanuella Scott, 1901, Hase Corgosinho, Kihara, Schizas, Ostmann, Martinez Arbizu \& Ivanenko, 2018.

Body robust, dorso-ventrally compressed; first pedigerous somite incorporated into the cephalosome forming a carapace-like extension of the cephalosome in Cerviniella and Eucanuella (Fig. 3e) and completely separated in Hase (arrow in Fig. 3b). Rostrum small and fused to cephalic shield; A1 short, 5-7 segmented with many setae transformed into strong spines (arrow in Fig. 3f), A2 shortened, with complete or incomplete allobasis, exp. 4-segmented with setae formula 2.1.1.2 or 2.1.1.3 (Fig. 3f), apical elements of enp 2 transformed into strong spines (arrow in Fig. 3c). Maxillular epipodite absent or represented by one seta. P1-P4 ramus bent inwards (arrow in Fig. 3a, e); P2-P4 enp reduced (arrow in Fig. 3d), with a maximum of 2 segments per ramus, in many cases reduced to a single segment or absent; exp. with 1, 2 or 3 segments. Urosome with strong hook-like lateral projections on genital double somite in Cerviniella species (arrow in Fig. 3e, d) and on last
Urs in Hase (Fig. 3b). Furca divergent or parallel; not elongated, normally as long as anal somite or shorter; anterolateral accessory seta (I) inserted near to proximal margin, inserted at 10-30\% of total length of furca (arrow in Fig. 3e); anterolateral caudal seta (II) inserted at $20-50 \%$ of furca (arrow in Fig. 3e).

\section{Cerviniinae Sars. M. 1903}

Type genus: Cervinia Norman in Brady, 1878

Other genera: Expansicervinia Montagna, 1981; Paracerviniella Brodsky, 1963.

Body slightly elongated, with clear separation between prosome and urosome; first pedigerous somite free (arrow in Fig. 5a, b). Rostrum slightly produced and fused to cephalic shield; A1 approximately the same length as cephalosome, 6-8 segmented without spinelike seta, A2 with complete or incomplete allobasis, exp. 4-segmented with setae formula 2.1.1.3 in all species (Fig. 5c). Maxillular epipodite represented by one seta. P1-P4 rami in normal position; P1-P4 exp. 3-segmented, P1-P4 enp 2-3 segmented. Female P5 1-segmented (arrow in Fig. 5a). Urs somites without hook-like lateral projections. Furca divergent or parallel; not elongated, normally as long as or slightly longer than anal somite; anterolateral accessory seta (I) inserted near proximal margin (arrow in Fig. 5b), anterolateral caudal seta (II) inserted at posterior half of furca (arrow in Fig. 5b).

Remarks: the inclusion of the genus Paracerviniella within this family should be taken carefully, this taxon was described based only in a single male specimen. The variability and high dimorphism present in the Aegisthidae males makes difficult to decide the correct position of this taxon, the genus is temporally retained here until further specimens allowed a complete comparison.

\section{Cerviniopseinae Brotskaya, 1963}

Type genus: Cerviniopsis Sars, G. O. 1903.

Other genera: Aegisthidae gen. 2; Hemicervinia Lang, 1935, Herdmaniopsis Brotskaya, 1963; Siphonis MercadoSalas, Khodami \& Martínez Arbizu, 2019; Stratiopontotes Soyer, 1970; Tonpostratiotes Itô, 1982.

Body elongated typically with first pedigerous somite free (arrow in Fig. 4d), when fused to cephalosome the suture is always conspicuous. Rostrum triangular in shape and fused with cephalosome, in some species well developed. Prosome without ornamentation of elaborate dorsal processes (Fig. 4a, d); A1 5-8 segmented, setae slender and long (arrow in Fig. 4c) and without spinous processes on first four segments, first and second segments not elongated. A2 with complete or incomplete allobasis, free endopodal segment about the same size of allobasis; exp. 4-segmented with setae formula 2.1.1.2 or 1.1.1.2 (Fig. 4e). Basis of mandibular palp quadrated as long as wide or slightly wider, enp and exp-1 not 
elongated (arrow in Fig. 4a). Maxillular epipodite represented by one seta (in some species absent). P1-P4 rami straight (not bended), usually with elongated exp. and enp (Fig. 4b, e); P1-P4 enp and exp. 3-segmented (members of Hemicervinia and Aegisthidae gen. 2 with P1 enp 2 -segmented). Furca adpressed (parallel) along entire inner margin (arrow in Fig. 4b, e), usually about same size or slightly longer than total length of urosome; anterolateral accessory seta (I) inserted at $20-50 \%$ of total length of furca (arrow in Fig. 4a); anterolateral furcal seta (II) inserted at $80-90 \%$ of furca (arrow in Fig. 4a).

\section{Aegisthinae Giesbrecht, 1893}

Type genus: Aegisthus Giesbrecht, 1891

Other genera: Andromastax Conroy-Dalton \& Huys, 1999; Jamstecia Lee W. \& Huys, 2000; Nudivorax Lee W. \& Huys, 2000; Scabrantenna Lee W. \& Huys, 2000.

Body elongated, with first pedigerous somite free. Rostrum fused to cephalosome (arrow in Fig. 6b), in some species not prominent but in other well developed (especially in Aegisthus). Prosome with or without ornamentation of elaborate processes (arrow in Fig. 6b); A1 6-7 segmented, with or without spinous processes (arrow in Fig. 6b); A2 with complete allobasis bearing only one small seta; exp 2-3 segmented (Fig. 6c). Mandibular palp reduced to 1-2 segments bearing 2 setae (arrow in Fig. 6c); maxillular epipodite and exopodite absent; Mxp reduced to 2-3 segments (arrow in Fig. 6c); males with more reduced mouthparts. P1 enp 2segmented in all genera (suture between enp2 and enp3 usually conspicuous) with exception of Aegisthus where it retains 3-segmented state; P1-P4 exp. always 3segmented, rami usually elongated. Furca adpressed (parallel) along entire margin (arrow Fig. 6a), slightly longer than total length of urosome in the genus Nudivorax or longer than the body in the other genera. Anteroraleral accessory seta (I) reduced or absent, when present always inserted at $20-30 \%$ of total length of furca; anterolateral caudal seta (II) always present and inserted at $50 \%$ of furca.

\section{Pontostratiotinae Scott, A. 1909}

Type taxon: Pontostratiotes Brady, 1883

Body elongated, with first pedigerous somite free. Rostrum triangular and fused to cephalosome, not well developed. Usually with elaborated dorsal process on the prosome (arrow in Fig. 6e), some members without. A1 8-segmented, spinous process on segments 1-2 always present (arrow in Fig. 6d, e) (some species with additional processes on segments 3-4); segments 1-3 elongated. A2 with complete or incomplete allobasis; free endopodal segment elongated, usually longer than allobasis; exp. 4-segmented with setae formula 2.1.1.2 or 0.1.1.2. Basis of mandibular palp rectangular, always longer than wide; enp and exp-1 elongated, at least three times longer than wide and bent outwards, being almost parallel to basis. Maxillular epipodite represented by one seta (in some species absent). Mx2 and Mxp following Aegisthidae ground pattern (arrow in Fig.6f). P1-P4 rami straight; enp and exp. elongated and 3 -segmented. Furca adpressed (parallel) along entire margin (arrow in Fig. 6d); usually as long as or longer than total body length (in some species rami as long as urosome); anterolateral accessory seta (I) inserted at $20-30 \%$ of total length of furca (arrow Fig. $6 \mathrm{~d}$ ); anterolateral caudal seta (II) inserted at $60-90 \%$ of furca (arrow Fig. 6d).

\section{Supplementary information}

Supplementary information accompanies this paper at https://doi.org/10. 1186/s12862-020-1594-X.

Additional file 1. The second tree topology.

Additional file 2. MrBayes Job1.

Additional file 3. MrBayes Job2.

Abbreviations

18 rRNA: Small subunit 18 ribosomal RNA; 285 rRNA: Large subunit 285 ribosomal RNA; A1: Antennule; A2: Antenna; COI mtDNA: Cytochrome c oxidase subunit I mitochondrial DNA; Enp: Endopod; Exp: Exopod; Md: Mandible; Mx1: Maxillula; Mx2: Maxilla; Mxp: Maxilliped; P1-P6: First to sixth thoracopod; Urs: Urosomite(s)

\section{Acknowledgements}

We thank Saskia Brix and Angelika Brandt for inviting us to join the IceAGE ME 85-3 and Kurambio-II cruise. We thank the captain and crew members of the research vessels SONNE and METEOR. We are grateful to Terue Cristina Kihara for her kind helps and advices to identify the aegisthid species used in this study. This publication has the number 62 from the Senckenberg am Meer Metabarcoding and Molecular Laboratory and number 48 that uses data from the Senckenberg am Meer Confocal Laser-scanning Microscope Facility.

\section{Authors' contributions}

All authors committed fairly for sampling and processing the samples. DNA laboratory works was conducted by SK and NM. Genetic sequence data and phylogenetic analyses were performed by SK and PM. The morphological identification of the copepod species was carried out by NM and PM. The manuscript is drafted by SK and the systematic part is written by NM. All authors contributed to the discussion and revised the manuscript based on their expertise and approved the manuscript prior submitting.

\section{Funding}

The SO250 "Kurambio-II cruise" was funded by grant number 03G0250B and SO239 by grant 03F0707E. Both grunt are funded and approved by the European Union Seventh Framework Program FP7/2007-2013. The addressed funding was provided to cover the expenses of the research vessels during sampling period. The funding bodies played no role in the design of the study and collection, analysis, and interpretation of data and in writing the manuscript.

\section{Availability of data and materials}

The datasets generated and analyzed during the current study including sequences alignment, nexus blocks used for MrBayes phylogenetic analyses, generated trees and the MrBayes log files (information about the runs) are available as supplementary information file 1 and 2 and all sequences are available in GenBank following the accession numbers MN536817 MN536902 for 185 rRNA, MN536171 - MN536215 for COI mtDNA and MN535552 - MN535623 for 28S rRNA. 


\section{Ethics approval and consent to participate}

Not applicable.

\section{Consent for publication}

Not applicable.

\section{Competing interests}

The authors declare that they have no competing interests.

Received: 10 October 2018 Accepted: 5 February 2020

Published online: 14 March 2020

\section{References}

1. Kabata Z. Parasitic Copepoda of British fishes. London: The Ray Society; 1979.

2. Marcotte BM. Evolution within the Crustacean. Part 2. Copepoda. In: Abele LG, The Biology of Crustacea. 1. Systematics, the Fossil Record and Biogeography, London Academic; 1982. p. 185-197.

3. Marcotte BM. Behaviorally defined ecological resources and speciation in Tisbe (Copepoda: Harpacticoida). J. Crustacean Biol 1984; 4404.

4. Marcotte BM. Phylogeny of the Copepoda Harpacticoida. Sylloge. 1986;58.

5. Bell SS, Hicks GRF, Walters K. Active swimming in meiobenthic copepods of seagrass beds: geographic comparisons of abundances and reproductive characteristics. Mar Biol. 1989:5:351.

6. Hicks GRF. Distribution and behavior of meiofaunal copepods inside and outside seagrass beds. Mar Ecol Prog Ser. 1986;31:159.

7. Hicks GRF. Systematics of the Donsiellinae Lang 1948 (Copepoda, Harpacticoida). J Nat Hist. 1988:22, 639.

8. Coull BC. Species diversity and faunal affinities of meiobenthic Copepoda in the deep sea. Mar Biol. 1972;14:48.

9. Brotskaya VA. A survey of the family Cerviniidae. Zoologicheskii Zhurnal SSSR; 1963. p. 42.

10. Huys R. Sexual dimorphism in aegisthid cephalosomic appendages (Copepoda, Harpacticoida): a reappraisal. Bijdragen tot de dierkunde. 1988; 58:114.

11. Huys R. Styracothoracidae (Copepoda: Harpacticoida), a new family from the Philippine deep sea. J. Crustacean Biol. 1993;13:769.

12. Montagna PA, Carey AG. Distributional notes on Harpacticoida (Crustacea: Copepoda) collected from the Beaufort Sea (Arctic Ocean). Astarte. 1978;11.

13. Por FD. A study of the Levantine and Pontic Harpacticoida (Crustacea, Copepoda). Zool Verh. 1964;64.

14. Por FD. Deep-sea Cerviniidae (Copepoda: Harpacticoida) from the western Indian Ocean, collected with RN Anton Bruun in 1964. Smithson. Contrib Zool 1969; 29

15. Shimanaga M, Nomaki H, lijima K. Spatial changes in the distributions of deep-sea Cerviniidae (Harpacticoida, Copepoda) and their associations with environmental factors in the bathyal zone around Sagami Bay. Japan Mar Biol. 2008;153.

16. Lang K. Monographie der Harpacticiden (vorläufige Mitteilung). Uppsala: Almquist and Wiksells Boktryckeri AB; 1994. p. 39.

17. Boxshall GA, Halsey SH. An Introduction to Copepod diversity. London: The Ray Society; 2004. p. 251-306.

18. Huys R, Møbjerg N, Kristensen RM. New tantulocarid Poynyapodella ambrosei gen. et sp. nov., (Basipodellidae) from the north east wate polynya (Greenland) with emphasis on the phylogeny of its host genus Cervinia (Copepoda: Harpacticoida). Cah Biol Mar. 1997;38:27.

19. Lee W, Huys R. New Aegisthidae (Copepoda: Harpacticoida) from western Pacific cold seeps and hydrothermal vents. Zool. J Linnean Soc 2000; 1291.

20. Park E, Shimanaga M, Yoon S, Lee W. A new species of the genus Cerviniopsis from Sagami Bay, Japan and reinstatement of the genus Neocervinia, with a report on the male of Neocervinia itoi Lee and Yoo, 1998 (Copepoda: Harpacticoida: Aegisthidae). Zootaxa. 2012:3575.

21. Seifried S. Phylogeny of Harpacticoida (Copepoda): revision of "Maxillipedasphalea" and Exanechentera. Göttingen: Cuvillier Verlag; 2003.

22. Seifried S, Schminke HK. Phylogenetic relationships at the base of Oligoarthra (Copepoda, Harpacticoida) with a new species as the cornerstone. Org Divers Evol. 2003;3.

23. Huys R, Mackenzie-Dodds J, Llewellyn-Hughes J. Cancrincolidae (Copepoda, Harpacticoida) associated with land crabs: a semiterrestrial leaf of the ameirid tree. Mol Phylo Evol. 2009;51:143.
24. Corgosinho PHC, Kihara TC, Schizas NC, Ostmann A, Martinez Arbizu P, Ivanenko VN. Traditional and confocal descriptions of a new genus and two new species of deep water Cerviniinae Sars, 1903 from the southern Atlantic and the Norwegian Sea: with a discussion on the use of digital media in taxonomy (Copepoda, Harpacticoida, Aegisthidae). ZooKeys. 2018;766:1.

25. Soyer J. Contribution à l'étude des Copépodes Harpacticoïdes de Méditerranée occidentale. I. Cerviniidae Sars, Lang. Vie. et. Milieu. 1970; 20.

26. Mercado-Salas NF, Khodami S, Martínez AP. Convergent evolution of mouthparts morphology between Siphonostomatoida and a new genus of deep-sea Aegisthidae Giesbrecht, 1893 (Copepoda: Harpacticoida). Mar Bio. 2019:49:1335-65.

27. Conroy-Dalton S, Huys R. A new genus of Aegisthidae (Copepoda, Harpacticoida) from hydrothermal vents on the Galapagos rift. J. Crustacean Biol. 1999;19:408.

28. Itô T. Harpacticoid copepods from the Pacific abyssal off Mindanao. I. Cerviniidae. J Fac Sci Hokkaido Univ. 1982:23.

29. Wells JBJ. An annotated checklist and keys to the species of Copepoda Harpacticoida (Crustacea). Zootaxa. 2007;1568

30. Huys R. Unresolved cases of type fixation, synonymy and homonymy in harpacticoid copepod nomenclature (Crustacea: Copepoda). Zootaxa. 2009;2183:1.

31. Lang K. Copepoda" Notodelphyoida" from the Swedish west-coast with an outline on the systematics of the copepods. Arkiv För Zoologi. 1948;40.

32. Huys $R$, lliffe TM. Novocriniidae, a new family of harpacticoid copepods from anchihaline caves in Belize. Zool Scripta. 1998;27:1.

33. Boxshall GA, Stocke JH, Sanchez E. A new species of Stephos Scott, 1892 (Copepoda: Calanoida) from an anchihaline lava pool on Lanzarote, Canary Islands. Stygologia. 1990;5:33

34. Shimanaga $M$, Lee $W$, Nomaki $H$, lijima K. Sex ratio and gut contents of the deep-sea harpacticoid Neocervinia and other cerviniids: a possibility of reduced foraging among males. J Crustacean Biol. 2009;29.

35. Coull BC. Meiobenthic Harpacticoida (Crustacea, Copepoda) from the deep sea off North Carolina I. the genera Hemimesochra Sars, Paranannopus Lang, and Cylindronannopus n. g. Trans Am Microsc Soc. 1973;92:185.

36. Montagna PA. Cervinia langi n. sp. and Pseudocervinia magna (Copepoda: Harpacticoida) from the Beaufort Sea (Alaska, U.S.A.). Trans. Am. Microsc. Soc. 1979; 98.

37. Noodt W. Ecology of the Copepoda. In: Hulugs, N. C. (ed.) Proceedings of the first international conference on meiofauna. Smithson Contr Zool. 1971; 76:97-102.

38. Montagna PA. Morphological adaptation in the deep-sea benthic harpacticoid copepod family Cerviniidae. Crustaceana. 1982;42.

39. Brix S, Bauernfeind W, Brenke N, Błażewicz M, Borges V, Buldt K, Cannon J, Díaz-Agras G, Fiege D, Fiorentino D, Haraldsdóttir S, Hoffmann S, Holst S, Huettmann F, Jeskulke K, Jennings R, Kocot K, Khodami S, Lucas R, Yasuhara M. Cruise report M85/3 IceAGE1 2011 with RV meteor; 2011.

40. Brandt A. Cruise report SO-250 KuramBio II (Kuril Kamchatka biodiversity studies). Zool. Museum Hamburg: University of Hamburg, Centre of Natural History (CeNak); 2016. https://doi.org/10.4126/FRL01-006401131.

41. Martínez Arbizu P, Haeckel M. RV SONNE Fahrtbericht / cruise report SO239: EcoResponse assessing the ecology, connectivity and resilience of Polymetallic nodule field systems, Balboa (Panama) - Manzanillo (Mexico,) GEOMAR Helmholtz-Zentrum für Ozeanforschung, Kiel. 2015.

42. Greinert J. RV SONNE Fahrtbericht / Cruise Report SO242-1: JPI OCEANS Ecological Aspects of Deep-Sea Mining, DISCOL Revisited, Guayaquil Guayaquil (Equador), 28.07-25.08.2015. Open Access. GEOMAR Report, N. Ser. 026. Kiel, Germany: GEOMAR Helmholtz-Zentrum für Ozeanforschung; 2015. https://doi.org/10.3289/GEOMAR_REP NS 262015.

43. Estoup A, Largiader CR, Perrot E, Chourrout D. Rapid one-tube DNA extraction for reliable PCR detection of fish polymorphic markers and transgenes. Mol Biol Biotech. 1996;5:295.

44. Hillis DM, Dixon MT. Ribosomal DNA: molecular evolution and phylogenetic inference. Q Rev Biol. 1991;66:411-53. doi. https://doi.org/10.1086/417338.

45. Hamby RK, Zimmer EA. Ribosomal RNA sequences for inferring phylogeny within the grass family (Poaceae). Plant Syst Evol. 1988;160:29-37.

46. Laakmann S, Gerdts G, Erler R, Knebelsberger T, Martínez Arbizu P, et al. Comparison of molecular species identification for North Sea calanoid copepods (Crustacea) using proteome fingerprints and DNA sequences. Mo Ecol Resour. 2013:862-76.

47. Ortman BD. DNA Barcoding the Medusozoa and Ctenophora. Ph.D. thesis. University of Connecticut, Storrs. 2008; p. 244

48. Folmer OM, Black W, Hoen R, Lutz R, Vrijenhoek R. DNA primers for amplification of mitochondrial cytochrome c oxidase subunit I from diverse metazoan invertebrates Mol. Mar Biol Biotech. 1994;3:294-9. 
49. Bucklin A, Ortman B, Jennings R, Nigro L, Sweetman C, Copley N, Sutton T, Wiebe P. "Rosetta Stone" for metazoan zooplankton: DNA barcode analysis of species diversity of the Sargasso Sea (Northwest Atlantic Ocean). Deep Sea Res II. 2010;57:2234-47.

50. Katoh K, Misawa K, Kuma Kl, Miyata T. MAFFT: a novel method for rapid multiple sequence alignment based on fast Fourier transform. Nucleic Acids Res. 2002. https://doi.org/10.1093/nar/gkf436.

51. Katoh $\mathrm{K}$, Toh H. Recent developments in the MAFFT multiple sequence alignment program. Brief Bioinform. 2008;9:286.

52. Khodami S, McArthur JV, Blanco-Bercial L, Martinez AP. Molecular phylogeny and revision of copepod orders (Crustacea: Copepoda). Sci Rep. 2017. https://doi.org/10.1038/s41598-017-06656-4.

53. Altekar G, Dwarkadas S, Huelsenbeck JP, Ronquist F. Parallel metropolis coupled Markov chain Monte Carlo for Bayesian phylogenetic inference. J Bioinf. 2004;20:407.

54. Ronquist F, Huelsenbeck JP. MrBayes 3: Bayesian phylogenetic inference under mixed models. J Bioinformatics. 2003;19.

55. Posada D. jModelTest: Phylogenetic Model Averaging. Mol Biol Evol. 2008. https://doi.org/10.1093/molbev/msn083.

56. Huys R, Boxshall GA. Copepod Evolution. The Ray Society London. 1991.

57. Schminke HK. The ubiquitous telson and deceptive furca. Crustaceana. 1976; 30:292-300.

\section{Publisher's Note}

Springer Nature remains neutral with regard to jurisdictional claims in published maps and institutional affiliations.

Ready to submit your research? Choose BMC and benefit from:

- fast, convenient online submission

- thorough peer review by experienced researchers in your field

- rapid publication on acceptance

- support for research data, including large and complex data types

- gold Open Access which fosters wider collaboration and increased citations

- maximum visibility for your research: over $100 \mathrm{M}$ website views per year

At BMC, research is always in progress.

Learn more biomedcentral.com/submissions 